\title{
Modification des caractéristiques d'une turbulence sous l'influence de particules solides en suspension
}

\author{
Teneur limite d'un écoulement en matériaux \\ transportés par suspension
}

\author{
par M. Bouvard \\ Docteur ès Sciences, Directeur de l'Ecole \\ Nationale Supérieure d'Hydraulique de Grenoble
}

\author{
el S. Petkovic \\ Docteur Ingénieur, \\ Ingénieur à l'Institut Jarvoslav-Cerni, Belgrade
}

\section{Introduction}

La plupart des auteurs qui ont étudié le phénomène de suspension des particules solides dans un milieu turbulent ont surtout considéré le problème de la répartition des particules sous l'action de la turbulence du milieu porteur, sans, en général, prendre en considération les modifications apportées par la suspension elle-même sur la turbulence, supposée par conséquent indépendante des matériaux en suspension.

Même en ne considérant que ce premier aspect, le problème est extrêmement complexe, ce qui justifie une approche progressive basée sur l'hypothèse a priori suivant laquelle le second effet serait d'importance réduite. Mais il semble que les phénomènes liés à l'influence des matériaux en suspension sur la turbulence soient extrêmement importants, et que seule cette influence puisse expliquer des faits expérimentaux courants, comme l'existence d'une concentration limite en matériaux, pour un courant porteur dont le mouvement moyen présenterait des caractéristiques bien définies, ou la réduction des pertes de charge dans un écoulement chargé de matériaux [13].

Aussi nous avons voulu profiter de l'existence au laboratoire d'une installation qui permettait d'aborder expérimentalement le second aspect dans des conditions relativement faciles pour procéder à une première étude le concernant.

Etat actuel du problème. Principaux objectifs de l'étude.

Le problème posé par la suspension des particules solides dans un écoulement turbulent appartient au domaine général des écoulements polyphasiques. Son importance est bien connue, car il comprend divers phénomènes naturels (trans- port des matériaux solides dans les rivières) et procédés techniques (hydrotransport dans les conduites). Aussi a-t-il fait l'objet de nombreuses recherches. En particulier les écoulements polyphasiques ont constitué un des sujets principaux du XII' congrès A.I.R.H. (Japon, 1969).

En dépit d'efforts considérables, les écoulements polyphasiques sont bien loin d'être totalement éclaircis, au point de vue théorique, même en ce qui concerne les problèmes de fond essentiels. Les différents auteurs apportent des réponses contradictoires à des phénomènes de base. D'après certains, la suspension des particules solides cause la décroissance de la turbulence de l'écoulement porteur (Barenblatt [5]), d'après les autres, par contre, les particules solides créent une turbulence supplémentaire aux dépens de l'énergie de l'écoulement moyen (Hino [9]). Au point de vue de la structure turbulente, ce sont d'après Soo [10], les petits tourbillons qui sont les plus influencés, tandis que, d'après quelques chercheurs soviétiques [11], c'est dans la zone des petits nombres d'ondes (grands tourbillons) que la courbe de densité spectrale est la plus modifiée.

A une époque plus récente, plusieurs essais d'une analyse plus exacte et plus profonde du phénomène sont apparus. On peut mentionner, parmi ces théories, le système général des équations fondamentales concernant l'écoulement turbulent en présence de particules solides en suspension, proposé par l'auteur soviétique Frankle [12]. En écrivant, pour les deux phases, l'équation de continuité, les équations dynamiques, les équations d'énergie de l'écoulement moyen et des fluctuations turbulentes, et les équations thermodynamiques, il arrive à un système de 20 équations contenant 39 inconnues.

Cette voie théoriquement rigoureuse ne permet pas d'espérer pour le moment un progrès réel dans la connaissance de ce problème. D'un autre côté, si on introduit trop de simplifications pour pouvoir mener le calcul jusqu'à des 
Les résultats ont complété ceux obtenus au fil chaud - dans une gamme où ceux-ci ne s'appliquaient plus et ont permis de confirmer certains aspects de l'interprétation que nous avons donnée des résultats expérimentaux.

\section{Mesure des caractéristiques de la turbulence en présence de particules: vitesse, spectres $d$ 'énergie}

\section{Mise au point de la méthode avec le protecteur.}

Nous disposions ainsi des résultats de mesures portant sur les caractéristiques de la turbulence en eau pure, et il s'agissait de déterminer comment elles allaient être modifiées, en présence de particules solides, les caractéristiques du mouvement de la plaque - rappelons qu'il est piloté par un système électronique qui assure notamment la constance de la fréquence de son mouvement alternatif - restant bien entendu les mêmes.

Une première question se posait évidemment: le protecteur ne va-t-il pas entraîner une erreur inadmissible dans la mesure des caractéristiques de l'agitation turbulente même en eau pure? Aussi une première étape des mesures consistait à déterminer son influence éventuelle sur les

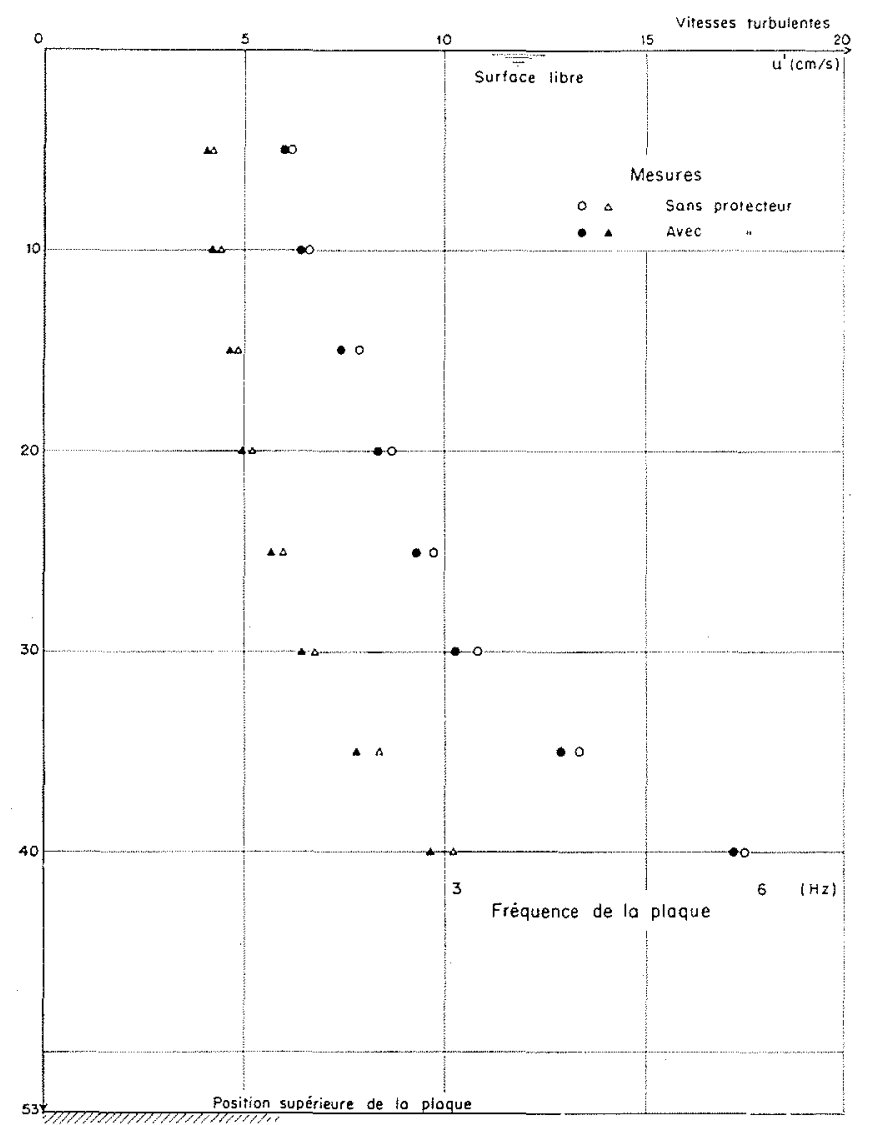

3/ Influence du protecteur de la sonde à film chand sur les mesures des vitesses turbulentes.

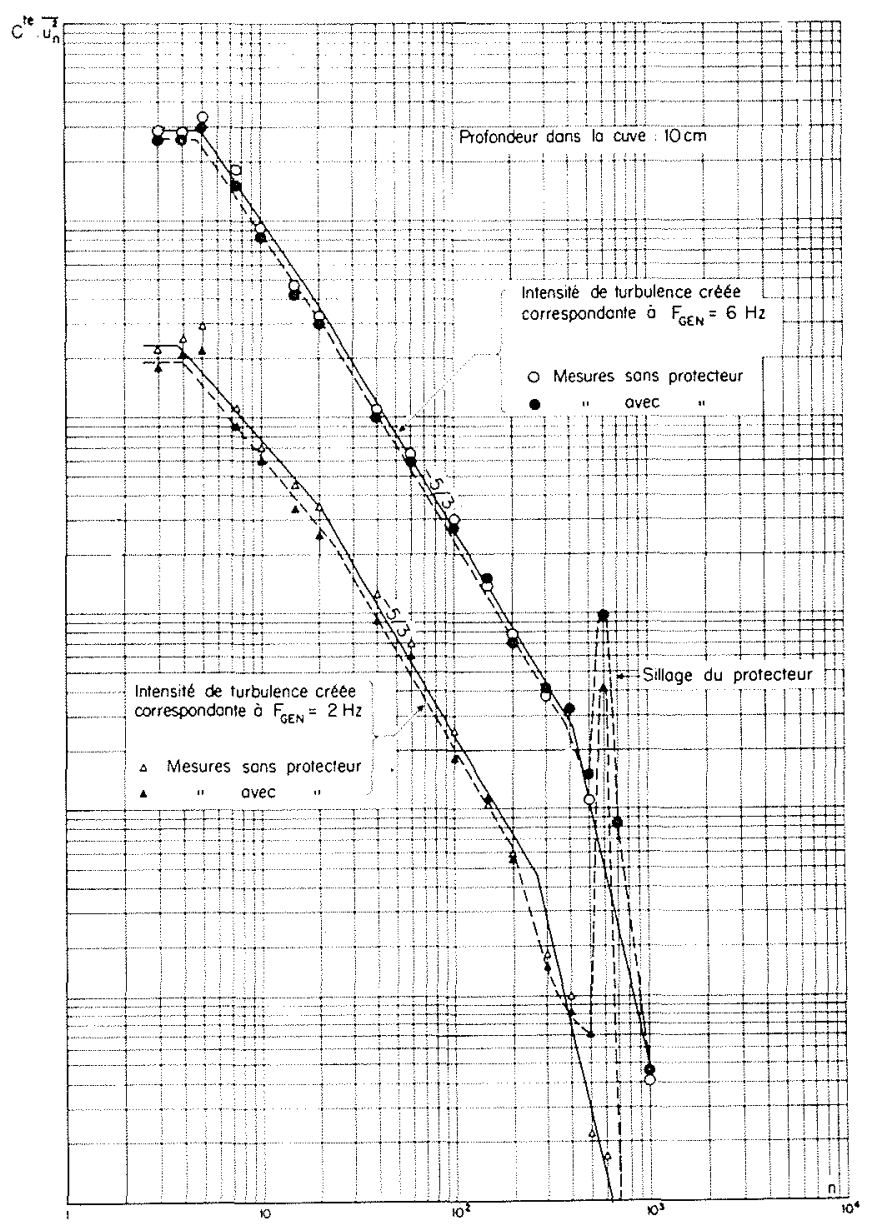

4/ Spectres d'énergie.

résultats. On a dans ce but effectué des mesures, avec et sans protecteur, aux différents niveaux de la cuve.

Les résultats sont présentés sur la figure 3. On peut constater que les valeurs de $u^{\prime}$, mesurées avec le protecteur, sont toujours inférieures aux valeurs correspondantes mesurées sans protecteur, les différences atteignant $5 \%$ environ. Bien entendu, ce résultat est fonction de la position de l'anneau de protection: il doit être placé de façon que le sillage qu'il crée atteigne aussi peu que possible l'élément sensible, donc le plus loin possible à l'amont de celui-ci. La limite correspond à la position suffisamment reculée pour qu'il assure juste - avec une certaine sécurité - sa fonction de protection.

Pour mieux éclaircir cet effet parasite, on a fait l'analyse spectrale des deux signaux correspondants, pour deux intensités de turbulence ( $F_{\mathrm{GEN}}$ égal à 2 puis à $6 \mathrm{hz}$ ). On constate que, sur presque toute la gamme des fréquences, son effet est négligeable, sauf pour les fréquences qui correspondent aux valeurs propres du sillage (fig. 4).

D'ailleurs, pour la plupart des mesures, même ces écarts n'entrent pas en ligne de compte: Lorsque nous avons effectué les mesures définitives dans l'eau pure, sans particules, nous avions placé le protecteur - bien qu'il soit 


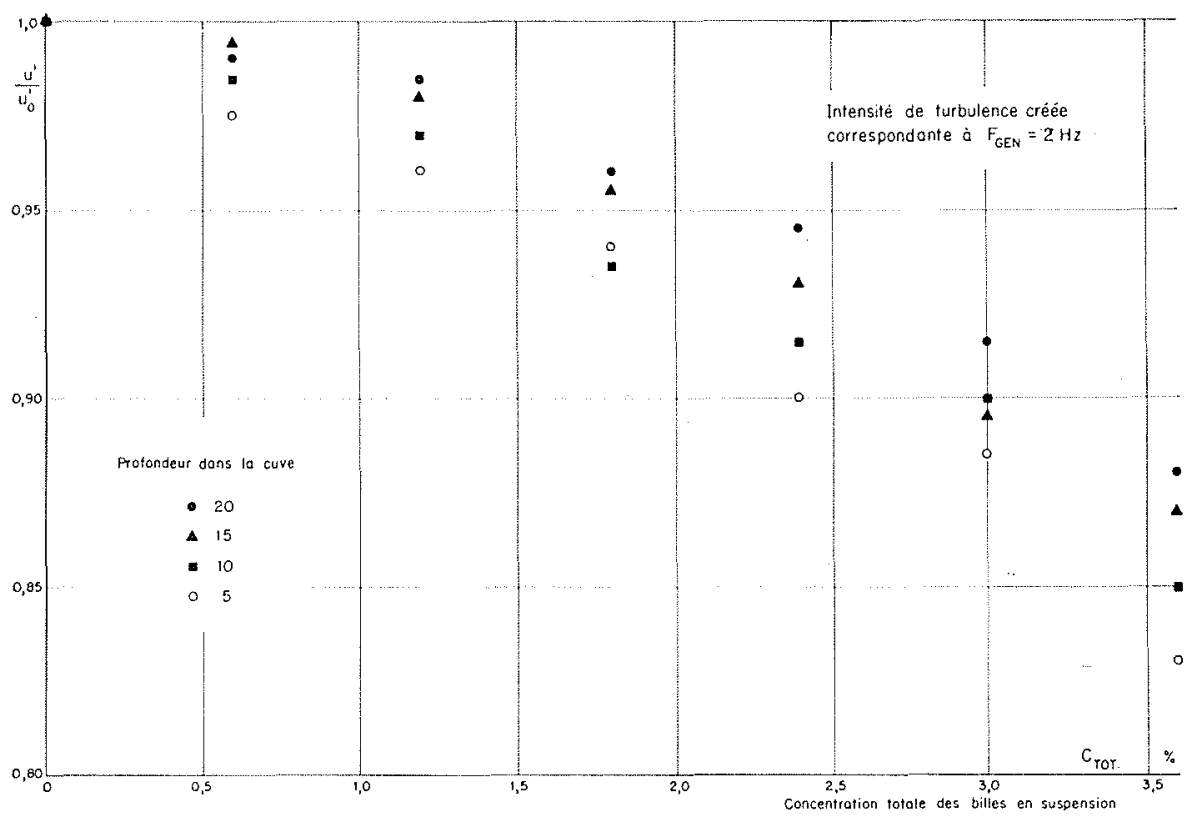

5/ Réduction de turbulence

par rapport à l'eau pure en présence

des particules solides

en suspension.

alors inutile - pour disposer de mesures qui soient placées le plus possible dans des conditions analogues.

Nous avons indiqué la fonction que devait remplir le dispositif électronique, lors de l'apparition du signal dû à l'influence des matériaux solides sur l'élément sensible: Comme on pourrait s'en douter, on constate que ce système fonctionne correctement quand la concentration en matériaux n'est pas trop élevée - sinon, le signal est constamment dévié.

Aussi nous avons limité les mesures de certaines caractéristiques du mouvement aux faibles profondeurs, et aux fréquences du mouvement générateur relativement réduites ( 2 et $3 \mathrm{hz}$ ). Dans ce cas, la concentration en particule est forte au fond, et faible ou très faible dans les parties supérieures de la cuve.

Par contre, les mesures au capteur de pression ne sont pas soumises à cette restriction, puisque le système est totalement insensible aux heurts des matériaux en suspension.

Résultat des mesures.

a) Vitesse.

Les résultats des mesures de $u^{\prime}$ sont présentés sur les figures 5 et 6 , en fonction de la concentration en particules et de la profondeur (limitée à des valeurs pas trop grandes).
Réduction de turbulence /6 par rapport à l'eau pure en présence des particules solides en suspension.

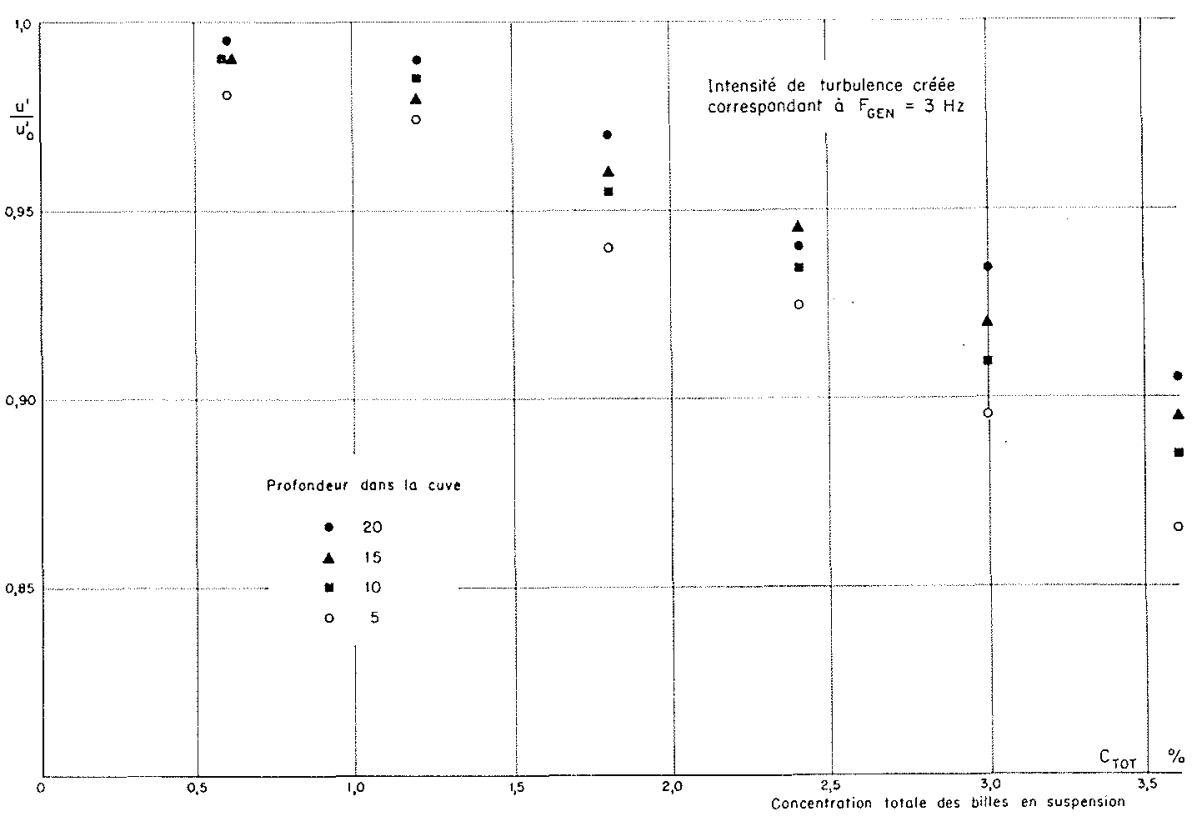


Oil note immédiatement que la vitesse turbulente diminue si on augmente la concentration. La réduction est d'ailleurs très sensible, puisqu'elle atteint 10 à $15 \%$ (correspondant à une réduction d'énergie turbulente de 20 à $30 \%$ ) pour une concentration moyenne relativement faible, égale à $3,6 \%$.

Il importe toutefois de remarquer que ces résultats concernent uniquement les cas où la concentration locale n'est pas trop élevée - pour les raisons indiquées ci-dessus. Le phénomène dans son ensemble est plus compliqué, et les mesures au capteur permettront d'en donner une interprétation plus complète.

\section{b) Spectres.}

La mesure de ceux-ci est toujours délicate, et nous avons considéré qu'il était impossible de l'exécuter avec le sys. tème électronique déviant le signal, lors des rencontres des billes avec l'élément sensible. La mesure a alors été limitée à la zone de la cuve dans laquelle les matériaux ne montaient pratiquement jamais, en limitant la fréquence du mouvement agitateur de la plaque à $2 \mathrm{hz}$. Une des mesures (fig. 7), a été réalisée sans le protecteur: il nous a semblé préférable de prendre le risque de casser l'élément sensible, lors d'un heurt, peu probable, avec une bille, pour rendre le résultat plus indiscutable.

On constate alors une différence, dans les spectres, lorsqu'il y a des billes dans la cuve: l'énergie correspondante aux basses fréquences est nettement diminuée. Pour les hautes fréquences, le résultat est inverse. L'énergie est alors augmentée, mais assez faiblement. Toutefois, la différence est absolument systématique, et tous les points correspondant aux mesures en présence de billes, entre 50 et $500 \mathrm{hz}$ environ, se situent au-dessus des points relevés par le mouvement en eau pure (fig. 7, 8, 9).

Ce sont toutefois les gros tourbillons qui restent les plus influencés.

c) Longueurs Caractéristiques: Micro échelle et ÉCHELLE INTÉGRALE.

Les spectres mesurés ci-dessus ont permis de calculer ces deux grandeurs. Compte tenu du déplacement des spectres vers les hautes fréquences, on constate qu'elles sont réduites en présence des billes, de $15 \%$ environ pour la micro échelle, et de $25 \%$ pour la longueur intégrale.

\section{Mesures de la concentration des particules en fonction de la quantité totale des billes en suspension}

\section{Procédé expérimental.}

Nous avons déjà mentionné le phénomène lié à la rencontre du protecteur et d'une bille en suspension : la vitesse au droit de l'élément sensible diminue brusquement, ce qui entraîne l'apparition de pics dans le signal sortie de l'anémomètre à température constante, correspondant à une baisse de la puissance nécessaire au maintien de l'élément sensible à la température de consigne.

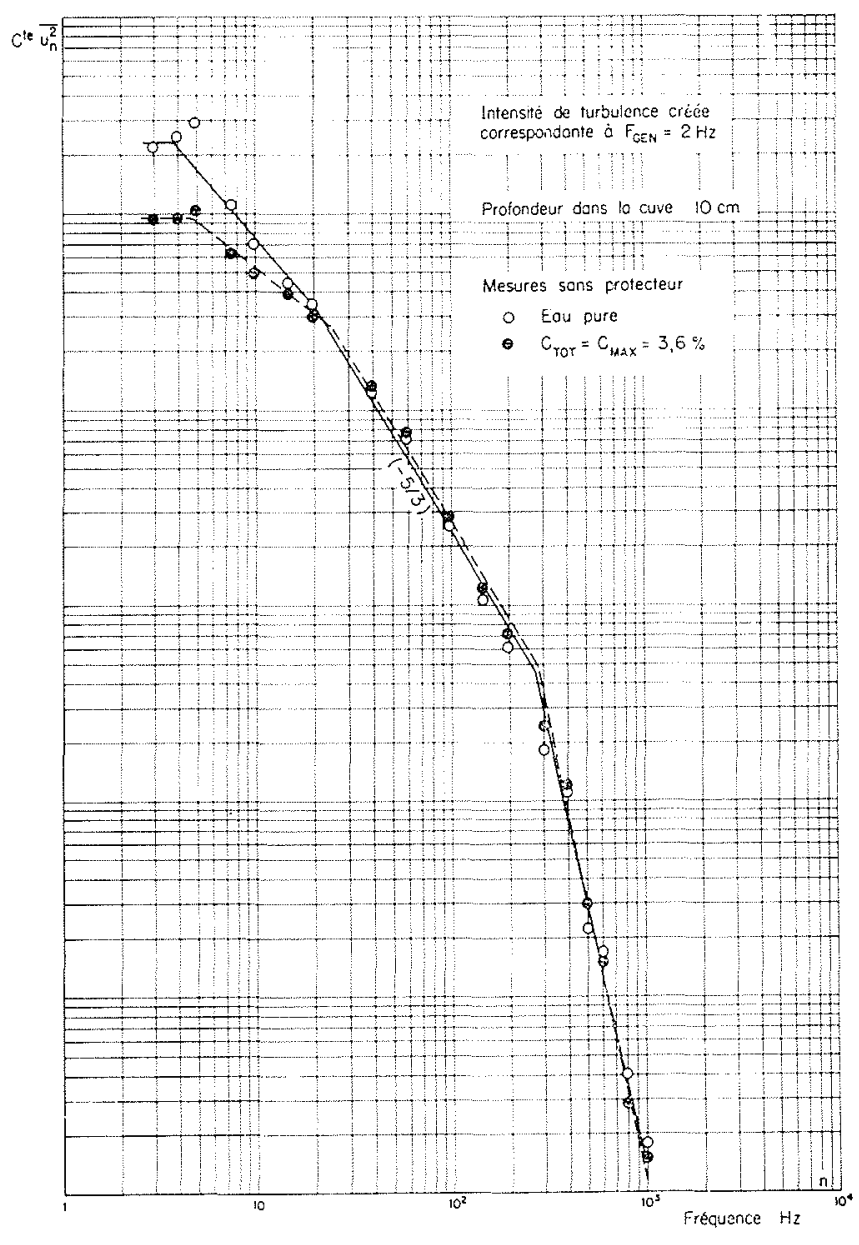

7/ Modification du spectre d'énergie en présence des particules solides en suspension.

Nous avons admis que la fréquence et la durée cumulée des pics parasites était proportionnelle à la concentration des particules au niveau considéré, caractérisé par le plan dans lequel se déplace le bras toumant.

C'est une hypothèse qui est tout à fait raisonnable, d'autant plus qu'il s'agit de mesures comparatives, à un niveau donné. Les vitesses d'agitation du fluide - qui, rappelonsle, diminuent avec le nombre total de particules dans la cuve - restent néanmoins d'un ordre de grandeur voisin, et les vitesses d'agitation des particules, à un niveau donné aussi. Comme la vitesse du bras tournant reste la même (elle est bien supérieure à la plus grande des vitesses turbulentes), les configurations de rencontre des billes avec le protecteur, suivant qu'elles bouchent totalement ou partiellement le protecteur, sont statistiquement les mêmes, et on conçoit très bien que, si on doublait la concentration en matériaux à niveau donné, on doublerait la durée des pics parasites. Chaque fois que nous avons eu l'occasion de vérifier cette hypothèse, nous avons trouvé qu'elle était confirmée.

On peut se demander seulement ce qu'elle deviendrait s'il s'agissait de procéder à des mesures de concentration relatives à deux niveaux différents, où les vitesses turbu- 


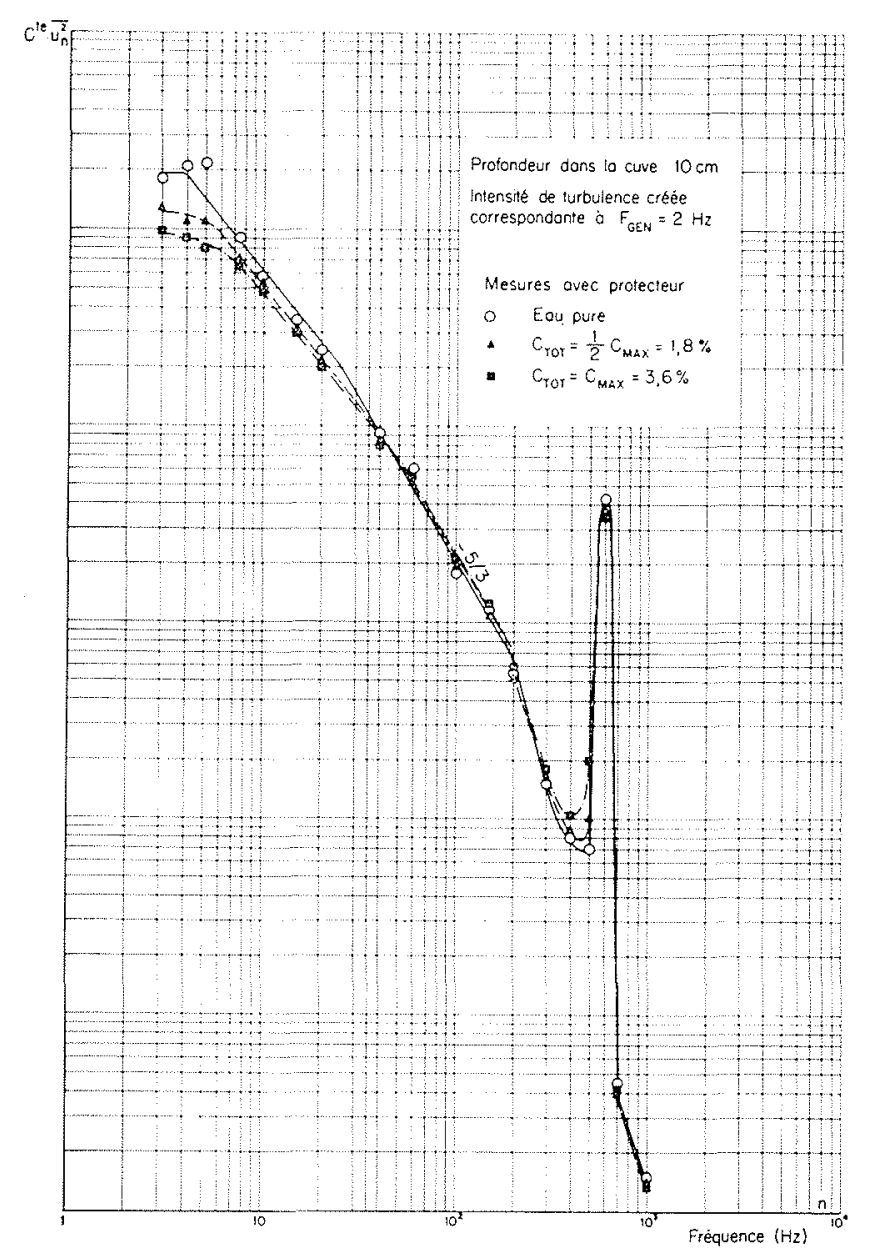

8/ Modification du spectre d'énergie en présence des particules solides en suspension.

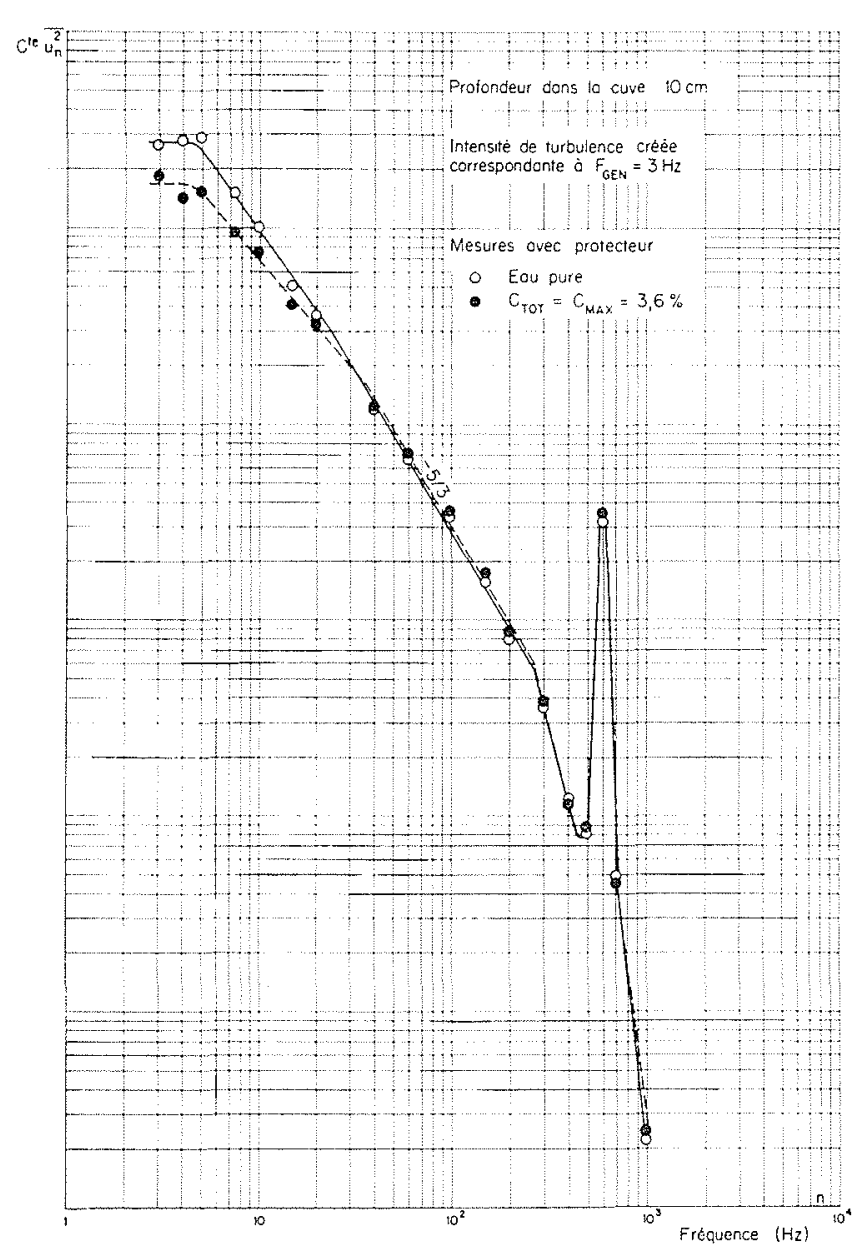

9/ Modification du spectre d'énergie en présence des particules solides en stuspension. lentes ne seraient pas les mêmes. Il nous paraît probable que l'hypothèse de base serait vérifiée, mais la question du réglage du seuil, examinée ci-dessous, poserait alors des problèmes. Aussi nous nous sommes limités à des comparaisons au même niveau, où la vitesse turbulente restait donc du même ordre de grandeur.

Le système électronique «coupait» donc les pics parasites au niveau d'un certain seuil, réglable, chacun d'eux ayant une durée propre $\Delta t$. Les tensions sorties de l'anémomètre à fil chaud était intégrées, sur une base de temps $T$, dans un voltmètre intégrateur (décrit dans [6]). L'élimination du signal pendant les durées $\Delta t$ faisait apparaître un temps $T^{\prime}=T-\Sigma \Delta t$ pendant la durée $T$ d'intégration du signal turbulent ou de son carré (pour avoir la valeur efficace).

Conformément à l'hypothèse ci-dessus, on peut considérer que l'indice $T=\Sigma \Delta t / T$ caractérise la concentration des billes à un niveau donné. Mais cette concentration dépend évidemment de la quantité totale des billes en suspension, dans la cuve, et croît à mesure que leur nombre augmente. Si $\mathrm{I}_{x}$ représente le résultat de la mesure pour $\mathrm{N}$ billes dans la cuve, le rapport $I_{N} / N$ caractérise la concentration relative, au niveau considéré. Pour de simples commodités d'écriture, nous n'avons pas pris pour $\mathrm{N}$ le nombre des billes, introduit dans la cuve, mais nous avons partagé le nombre total de celles à notre disposition (200 000 environ), en six unités égales, $\mathrm{N}$ représentant alors le nombre total d'unités introduites dans la cuve.

Nous avons alors déterminé la fonction $I_{N} / N$, pour différentes caractéristiques de l'intensité turbulente (suivant le mouvement de la plaque avec $F_{G \text { FN }}=2,3,4,5$ et $6 \mathrm{hz}$ ), différentes profondeurs dans la cuve $(10,20,30$ et $40 \mathrm{~cm})$, et bien entendu pour différentes valeurs de $\mathrm{N}$.

Il restait à régler le seuil de coupure du système électronique. Il ne pouvait être fixé à une valeur indépendante dc la profondeur, car l'intensité de l'agitation turbulente varie beaucoup en fonction de la profondeur: une valeur correcte pour les parties basses (où la vitesse turbulente est intense) aurait correspondu à un seuil beaucoup trop élevé pour les parties hautes. Aussi le réglage a-t-il été effectué pour chaque profondeur, avec le signal turbulent tel qu'il se présentait dans l'eau pure, de manière que le seuil se situât juste au voisinage du signal maximum sans le toucher. On devait alors obtenir $T^{\prime}=T$, c'est-à-dire que la lecture devait être la même, avec et sans intervention du système de coupure. 
Le réglage effectué, on introduisait les billes, en nombre croissant. On reprenait le réglage des seuils tel que réalisé ci-dessus, et on mesurait $T^{\prime}=T-\Sigma \Delta t$, pour chaque valeur des paramètres de base (profondeur, mouvement de la plaque, et nombre de billes). Certains essais en présence de particules ont même été effectués avec deux valeurs du seuil de coupure, le premier au voisinage de la valeur limite, le second plus éloigné, ce qui nous a permis de vérifier que les résultats obtenus restaient les mêmes. Les $I_{N}$ variaient bien entendu (en fonction de la valeur du seuil), mais les $I_{N} / N$ évoluaient de la même façon en fonction de $\mathrm{N}$.

\section{Résultat des mesures.}

Les résultats sont présentés sous forme de courbes donnant les valeurs de $I_{N} / N(*)$, en fonction des paramètres de base.

Mais nous croyons intéressant de rappeler au préalable la signification des courbes représentant :

$$
\frac{\mathrm{I}_{\mathrm{N}}}{\mathrm{N}} \frac{1}{\mathrm{I}_{\mathrm{N}=1}}
$$

a) Si les points expérimentaux sont rassemblés (avec une dispersion évidemment fonction de la précision des mesures) autour d'une droite horizontale cela signifie que la concentration relative ne change pas en fonction de la quantité totale des billes dans la cuve: Si, à la quantité totale des billes correspond une concentration à un niveau donné égale à $y$, il correspond à $2 y$ pour une quantité totale $2 x, 3 y$ pour $3 x$, etc.

b) Si les points expérimentaux montrent que le rapport $I_{N} / N$ diminue en fonction de $N$, cela signifie que la concentration relative est changée.

On a représenté sur les figures 12 et 15 deux cas extrêmes pour l'intensité de la turbulence créée dans la cuve: une forte turbulence $\left(\mathrm{F}_{\mathrm{GEN}}=6 \mathrm{hz}\right.$, fig. 12) et une faible turbulence ( $F_{\mathrm{GEX}}=2 \mathrm{hz}$, fig. 15).

On y constate les phénomènes suivants :

Dans le cas d'une forte intensité de la turbulence $\left(F_{\mathrm{GEN}}=5\right.$ et $6 \mathrm{hz}$, fig. 12 et 13$)$, la fonction $\mathrm{I}_{N} / \mathrm{N}$ est représentée par une droite horizontale. En fait, les valeurs trouvées sont un peu supérieures à l'unité, ce qui résulte, de même que la dispersion des points expérimentaux, du caractère aléatoire des grandeurs mesurées et de la précision des mesures.

(*) En fait, les courbes sont présentées en forme:

$$
\frac{\mathrm{I}_{\mathrm{N}}}{\mathrm{N}} \frac{1}{\mathrm{I}_{N=1}}=f(\mathrm{~N}) \text {. }
$$

Puisque le rapport $\mathrm{I}_{N} / \mathrm{N}$ est donné par un nombre quelconque dont la valeur absolue n'a pas de signification physique concrète, nous avons divisé tous les $I_{N} / N$, arbitrairement, par $\mathbf{I}_{\mathrm{N}=1} / 1$. Ainsi, si la concentration relative, dont l'indice est $I_{N} / N$, ne change pas en fonction de la quantité totale des billes en suspension (donné par $\mathrm{N}$ ), tous les points :

doivent être voisins de 1

$$
\frac{I_{N}}{N} \frac{1}{I_{N=1}}
$$

Dans le cas d'une faible intensité de la turbulence $\left(\mathrm{F}_{\mathrm{GEN}}=2,3 \mathrm{hz}\right.$ fig. 14,15 et 16$)$ la fonction :

$$
\frac{I_{N}}{N} \frac{1}{I_{N=1}}
$$

atteint très vite une valeur inférieure à l'unité.

On constate donc que, si l'intensité de la turbulence du mouvement de base est forte, la concentration à un niveau donné augmente proportionnellement à la quantité totale des billes en suspension dans la cuve (la concentration relative est constante), tandis qu'elle augmente moins que proportionnellement si l'intensité de la turbulence est faible (la concentration relative diminue avec le nombre de billes).

L'examen des photographies (figures 10 et 11) confirme tout à fait les données représentées par les courbes. Dans le cas de la figure 10, l'agitation turbulente est intense, et les particules sont réparties de façon homogène, qu'il y en ait beaucoup ou pas. Par contre, dans le cas de la figure 11 correspondant à une agitation plus réduite, on note immédiatement que la concentration relative n'est pas la même, suivant que les billes sont en plus ou moins grand nombre. S'il y en a beaucoup, elles restent concentrées vers le bas, alors qu'elles se répartissent plus uniformément s'il y en a peu.

\section{Interprétation des mesures.}

Ces résultats tendraient à prouver qu'il existerait une concentration limite, fonction des caractéristiques à la fois de la turbulence du courant porteur (caractérisée essentiellement par les paramètres classiques: notamment par la vitesse de fluctuation) et des matériaux en suspension (notamment: vitesse de chute en eau calme W).

En dessous de cette concentration limite, la concentration relative ne serait pas influencée par la charge en matériaux, tandis qu'elle décroîtrait au-dessus.

La fonction :

$$
\frac{I_{N}}{N} \frac{1}{I_{N=1}}
$$

aurait, en général, l'allure indiquée sur la figure 17 (a), avec deux valeurs limites, 1 et 0

Pour $\mathrm{C}<\mathrm{C}_{\mathrm{lim}}$, la fonction est représentée par la droite:

$$
\frac{I_{N}}{N} \frac{1}{I_{N=1}}=1
$$

Pour $\mathrm{C}>\mathrm{C}_{\mathrm{lim}}$, cette fonction décroîtrait en dessous de 1 pour atteindre rapidement une valeur nulle.

La quantité totale des billes en suspension dans la cuve (correspondant à une concentration moyenne de 3,6\%) représente donc, pour une forte turbulence de l'écoulement de base, une concentration inférieure à la concentration limite. La valeur de la fonction $I_{\mathrm{N}} / \mathrm{N}$ pour $\mathrm{F}_{\mathrm{GIN}}=5$ et $6 \mathrm{hz}$ représenterait donc la portion de la courbe générale située à gauche de la concentration limite.

Par contre, pour $\mathrm{F}_{\mathrm{GEN}}=2,3$ et $4 \mathrm{hz}$, la quantité totale de billes correspondrait à une concentration supérieure à sa valeur limite, pour les trois cas. Pour le cas extrême, $\left(\mathrm{F}_{\mathrm{GWN}}=2 \mathrm{hz}\right)$ la capacité de suspension (au niveau $-20 \mathrm{~cm}$ ) devient nulle dès que $N \geqslant 4$. On aurait dans ce cas la courbe $17(b)$ avec son allure générale, dans son ensemble. L'existence de cette concentration limite peut être ratta- 
chée au mécanisme, certainement très complexe, de l'interaction entre les particules solides et la turbulence de l'écoulement porteur. La répartition des particules solides en suspension dans un milieu turbulent dépend évidemment des caractéristiques de cette turbulence. On doit admettre que le rapport entre la vitesse turbulente $u^{\prime}$ (ou la composante verticale $v^{\prime}$, si la turbulence n'est pas isotrope) et la vitesse de chute en eau calme des particules solides joue dans ce phénomène un rôle essentiel. La concentration serait d'autant plus uniforme que $\mathrm{W}<u^{\prime}$. Mais les particules réagissent à leur tour sur l'écoulement porteur, provoquent une certaine modification de la turbulence (dans le sens d'une diminution des vitesses, et des caractéristiques de longueur de cette turbulence) qui, à son tour, changerait la répartition des concentrations, Aussi, on peut affirmer que, quand le rapport $I_{y} / N$ varie, c'est que la turbulence du milieu porteur a été modifiée.

Si on ajoute des matériaux dans un écoulement porteur, on peut admettre que le processus d'interaction entre les particules solides et la turbulence dure jusqu'au moment de la stabilisation du phénomène: La turbulence se réduirait jusqu'à atteindre des caractéristiques justes suffisantes pour maintenir en suspension les matériaux, lorsque la concentration limite serait atteinte. Au-delà, les capacités du courant porteur diminueraient, entraînant le dépôt des particules en excédent, et le phénomène se stabiliserait.

Ainsi, on pourrait définir la concentration limite des particules solides en suspension dans l'écoulement, comme la concentration dont l'effet sur la turbulence, par rapport aux forces qui accompagnent le mouvement turbulent du fluide, est à la limite pour laquelle la turbulence n'est pas encore significativement affectée.

\section{Essai d'analyse \\ du mécanisme des modifications apportées par les matériqux en suspension sur les caractéristiques de la turbulence}

\section{Bilan d'énergie.}

Ce phénomène a fait l'objet d'une étude de Barenblatt [5], l'un des premiers auteurs qui ait pris en considération la réaction de la suspension des particules solides sur la turbulence de l'écoulement porteur.
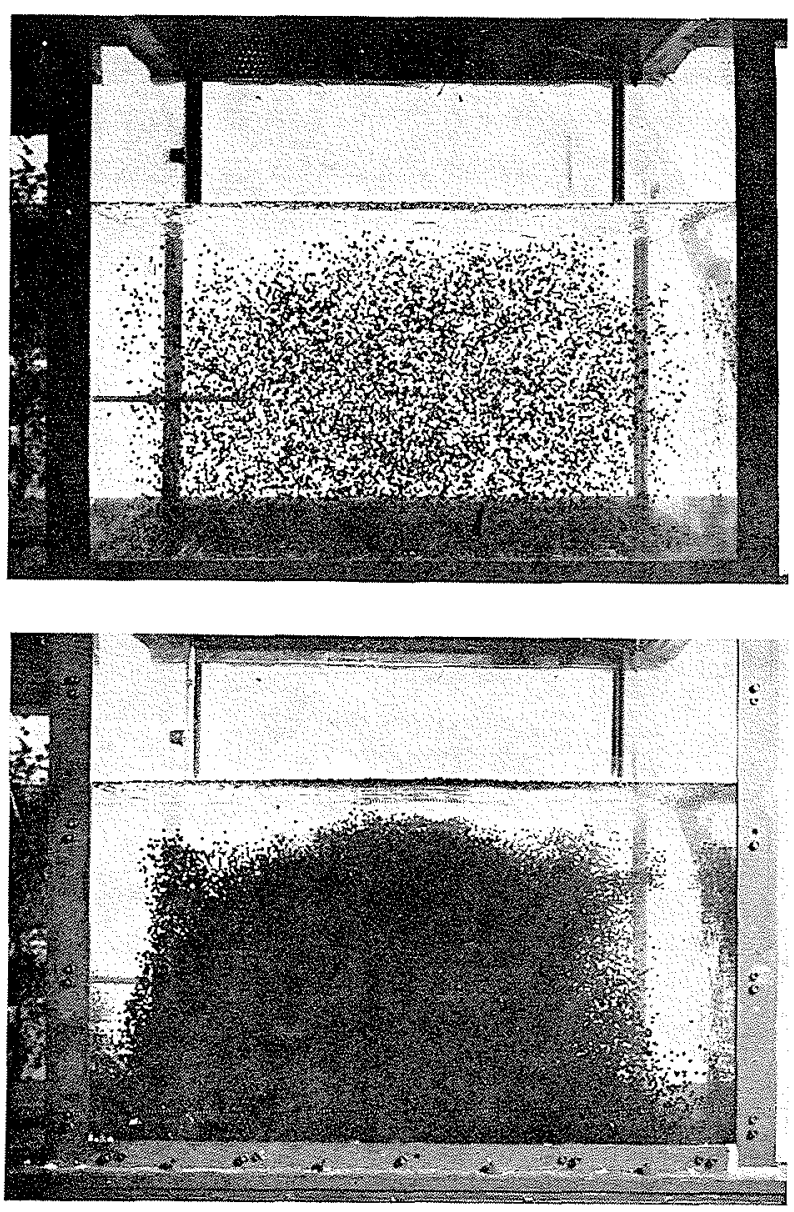

10/ Vue de la suspension dans la cuve dans les cas de faible et forte concentration des billes. (Intensité de turbulence créée correspondante à $F_{G, E N}=6 \mathrm{hz}$ ).
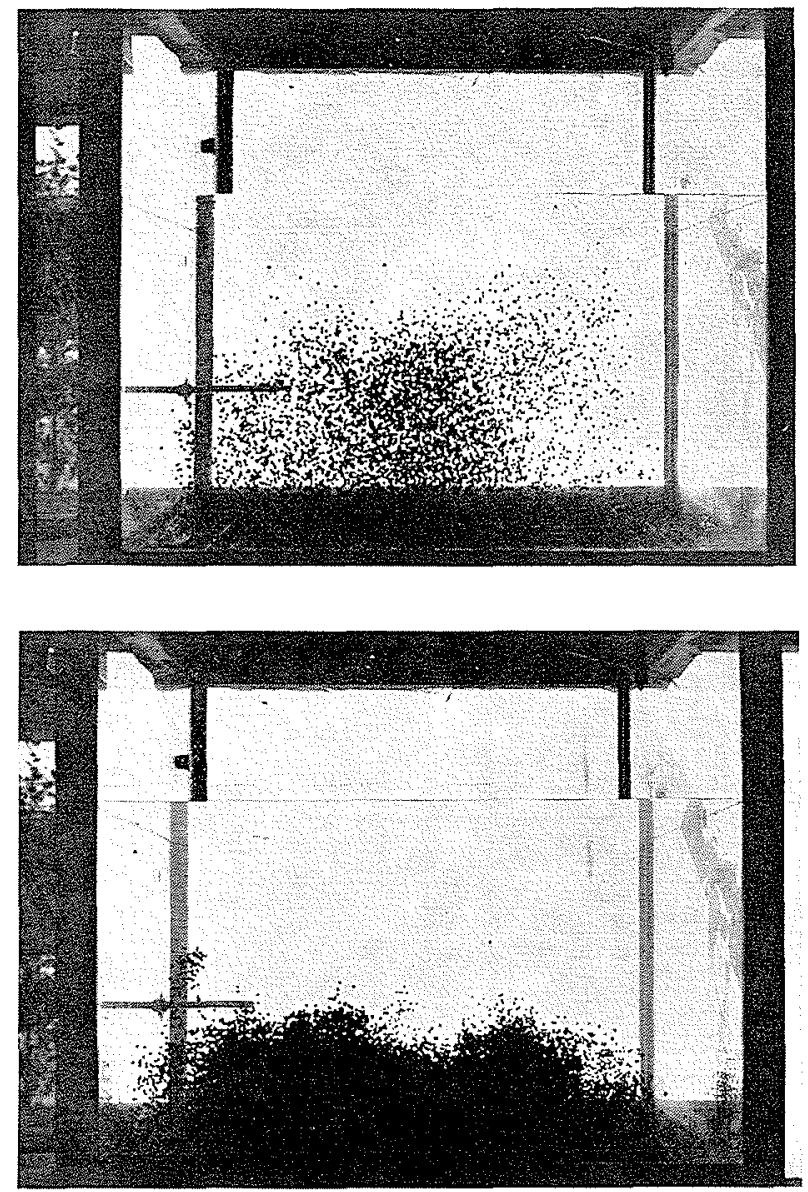

11/ Vue de la suspension dans la cuve dans les cas de faible et forte concentration des billes. (Intensité de turbulence créée correspondante à $\mathrm{F}_{\mathrm{GBN}}=2 \mathrm{hz}$ ) 


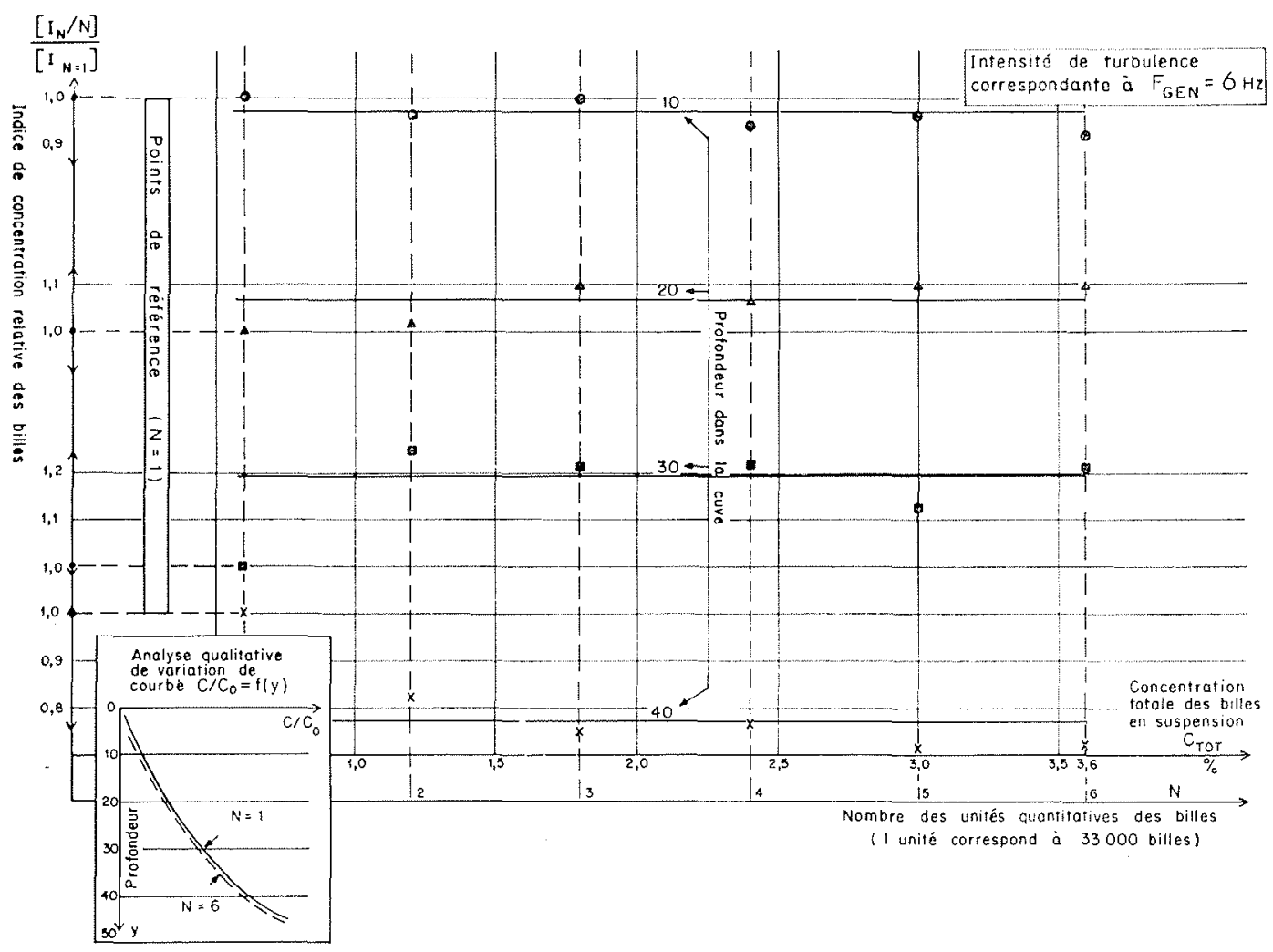

$12 /$

Variation de concentration

relative des billes

à un niveau donné en fonction

de la quantité totale

des billes en suspension.

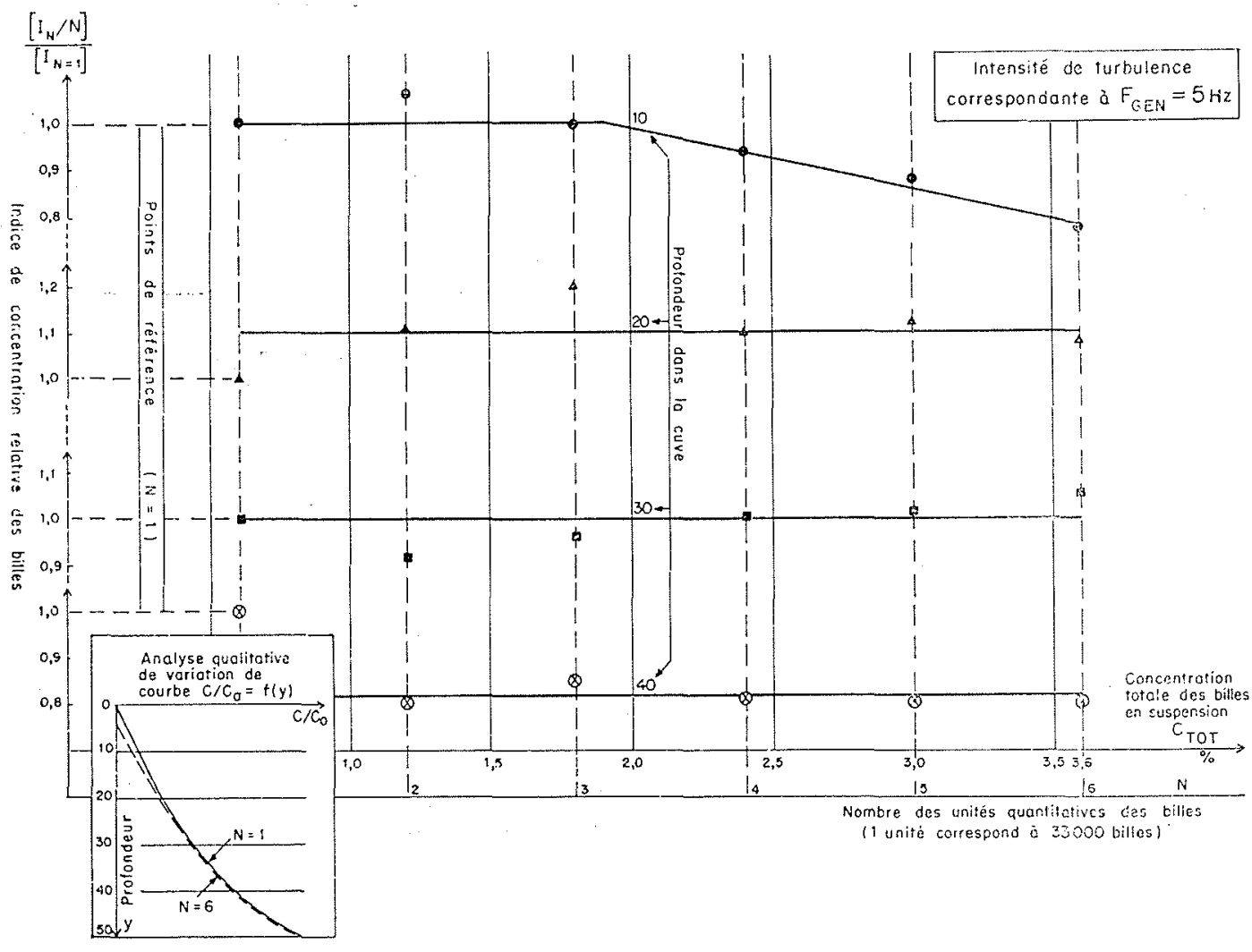

13/

Variation de concentration

relative des billes

à un niveau donné en fonction de la quantité totale

des billes en suspension. 

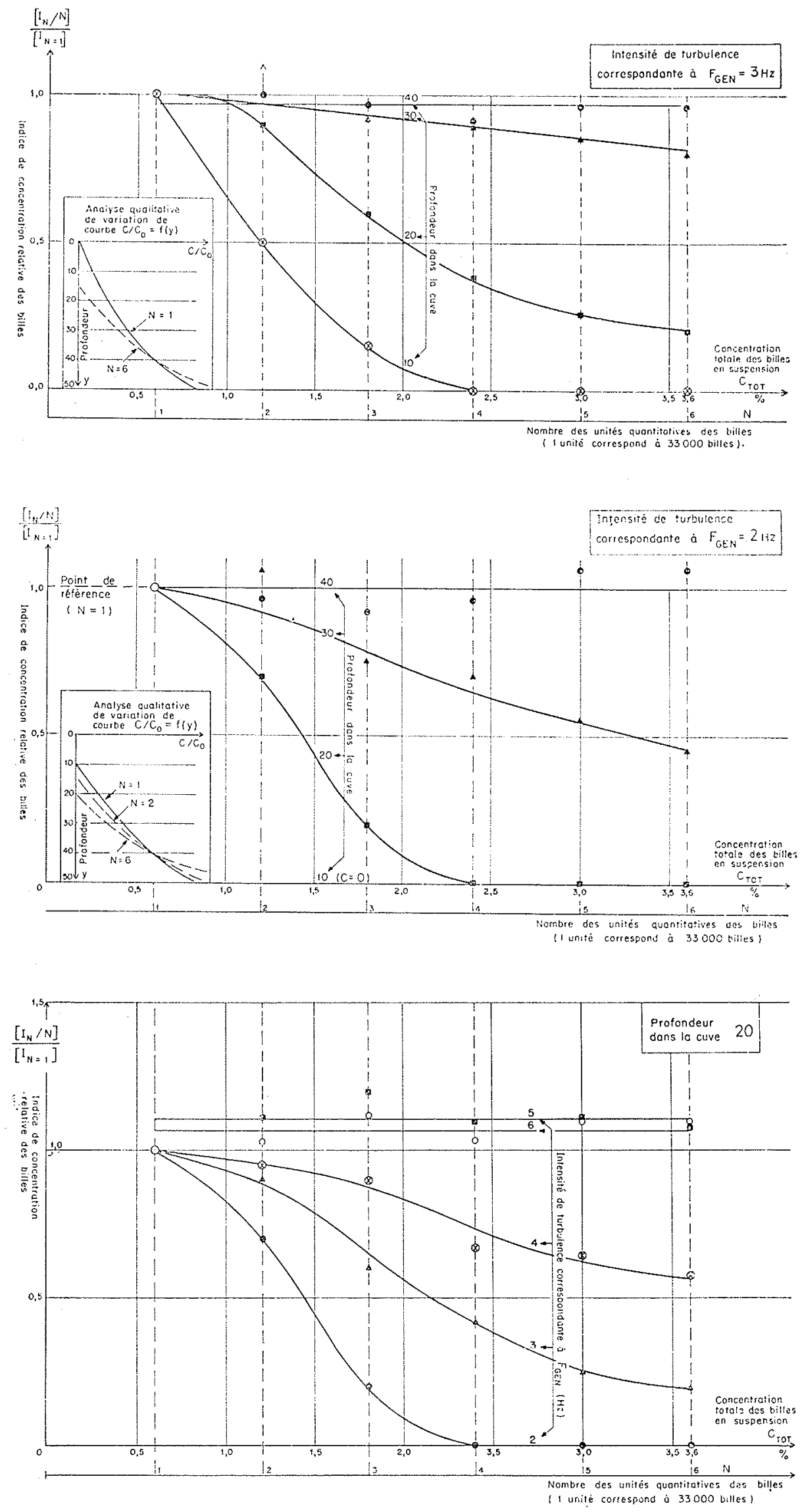

\section{$14 /$}

Variation de concentration

relative des billes

à un niveau donné en fonction de la quantité totale

des billes en suspension.

15/

Variation de concentration relative des billes à un niveatu donné en fonction de la quantité totale des billes en suspension.

$16 /$

Variation de concentration relative des billes

à un niveau donné en fonction de la quantité totale des billes en suspension. 


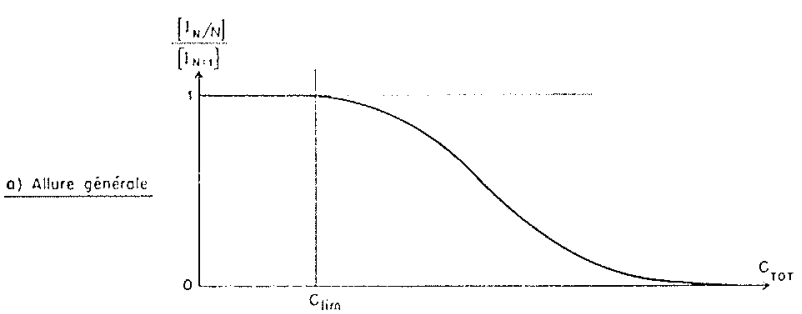

$c_{\text {lifi }}-$ Concentration limite

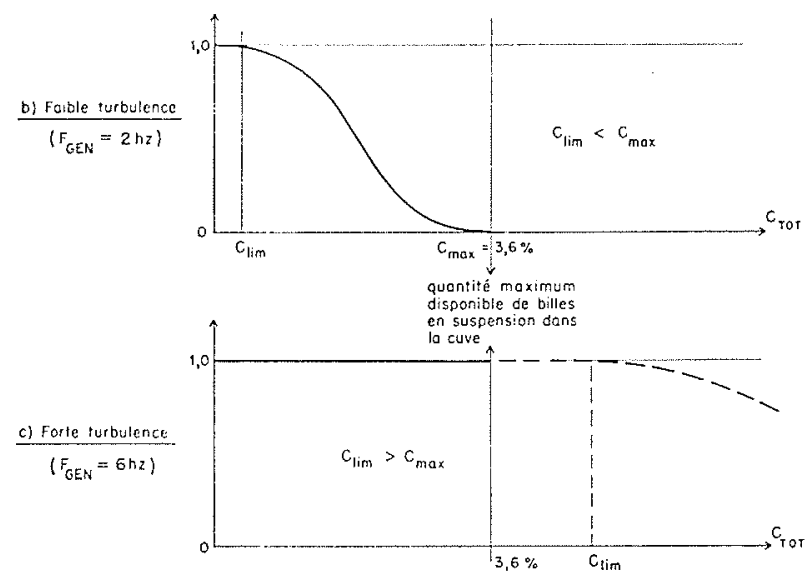

17/ Allure de la courbe $\left(\mathrm{I}_{\mathrm{K}} / \mathrm{N}\right) /\left(\mathbf{I}_{\mathrm{K}}=1\right)=f\left(\mathrm{C}_{\mathrm{TON}}\right)$.

Suivant la conception de Barenblatt, les modifications de la turbulence sous l'effet des particules dépend du rapport entre l'énergie fournie par l'écoulement pour mettre les particules en suspension et les autres termes du bilan d'énergie turbulente, avec, en premier lieu, la production d'énergie et la dissipation visqueuse.

Nous sommes arrivés à une conception analogue, mais d'aspect dynamique : les modifications dans la structure de la turbulence dépend du rapport entre «les efforts de réaction » des particules solides sur le mouvement porteur, et les efforts (dûs aux pressions) qui accompagnent le mouvement du fluide. Le concept est un peu différent de celui de Barenblatt. Il assimile l'effet global des particules sur la turbulence au «travail de mise en suspension», qui pourrait bien n'en représenter qu'un des aspects.

Par contre, nous considérons ici que l'effet est représenté par une «réaction des particules solides sur la turbulence», plus globale que celle envisagée par Barenblatt.

Nous pouvons essayer d'éclaircir la question traitée en considérant nos résultats expérimentaux.

Le bilan d'énergie turbulente a été établi pour le mouvement de l'eau pure dans la cuve, en écrivant que l'apport d'énergie turbulente par diffusion, à partir du fond de la cuve, est équilibré par la dissipation visqueuse, puisque le mouvement est statistiquement permanent [6].

Ces considérations se traduisent par l'équation:

$$
\frac{3}{2} \varepsilon_{0} \frac{d^{2} \mathrm{E}_{0}}{d y^{2}}=15 \vee \frac{\mathrm{E}_{0}}{\lambda^{2}}
$$

avec les notations suivantes:

$\varepsilon_{0}$ : coefficient de diffusion du mouvement turbulent;

$\lambda_{0}$ : micro échelle;

$\mathrm{E}_{0}: u_{0}{ }^{2}$, soit les $2 / 3$ de l'énergie cinétique turbulęnte, en considérant le mouvement comme isotrope.

L'indice $« 0 »$ souligne qu'il s'agit de grandeurs corres pondant au mouvement dans l'eau pure.

Suivant [6], les résultats de cette mise en équation cadrent bien avec le résultat des mesures.

Dans le cas des particules solides en suspension, nous pourrions mettre le bilan énergétique sous la forme suivante :

$$
\frac{3}{2} \varepsilon_{1} \frac{d^{2} \mathrm{E}_{1}}{d y^{2}}=15 \vee \frac{\mathrm{E}_{1}}{\lambda^{2}}+\Delta
$$

qui revient à admettre que toutes les grandeurs sont modifiées, tandis qu'on a introduit dans le bilan le terme supplémentaire $\Delta$ qui exprimerait, si on savait l'expliciter, la dissipation d'énergie correspondant à la présence des billes. $\Delta$ est représenté, en général, par l'énergie de mise en suspension des billes, et par la dissipation supplémentaire due au mouvement relatif de celles-ci par rapport à l'eau. On peut admettre que ce terme dépend de la concentration en particules solides, du rapport des densités des billes et du fluide, du rapport entre le diamètre des billes et la taille des plus petits tourbillons:

$$
\Delta=f\left(\mathrm{C}, \rho_{\mathrm{s}} / \rho_{\mathrm{f}}, d / \eta, \ldots\right)
$$

Considérons le rapport entre $\Delta$ et $15 \nu E_{1} / \lambda^{2}$, qui représentent les deux termes de «dissipation d'énergie». Si on considère les caractéristiques des deux mouvements d'agitation turbulente - une forte et une faible turbulence la différence des valeurs de la dissipation visqueuse est considérable. Pour une forte turbulence, E est plus grand et $\lambda$ plus petit.

Par contre, l'effet sur $\Delta$ doit beaucoup moins varier, à quantité totale de billes données (cas de notre étude expérimentale).

Essayons d'évaluer numériquement le rapport entre l'effet des particules solides sur la turbulence et la dissipation visqueuse dans le cadre de nos résultats expérimentaux.

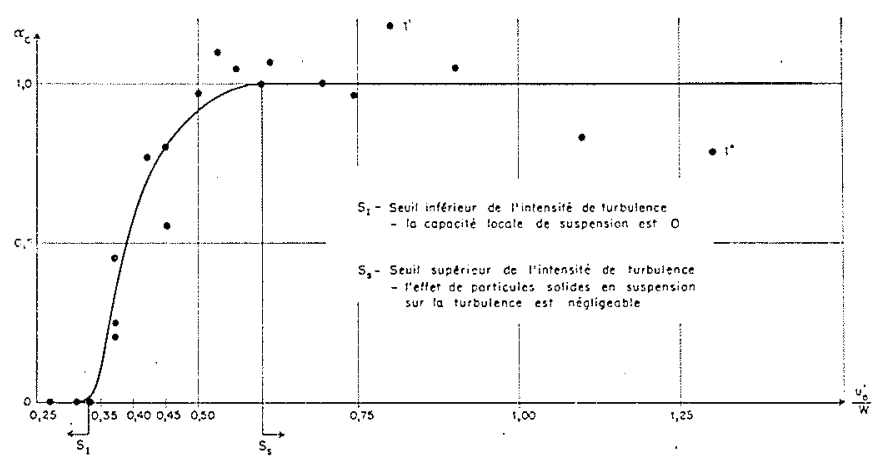

18/ Relation entre $\alpha_{c}=\left(I_{\sigma} / 6\right) / I_{1}$ à un niveau donné et la vitesse turbulente correspondante. 
Pour évaluer l'ordre de grandeur de $\Delta$, utilisons la formule de Barenblatt (*) [5] :

$$
\Delta=g W \sigma C \text {. }
$$

$\Delta$ est dimensionnellement homogène à une énergie par unité de masse, dans l'unité de temps. Dans notre cas, nous avons :

$$
\begin{gathered}
W=13,5 \mathrm{~cm} / \mathrm{s} \\
\sigma=\left(\rho_{s}-\rho_{f}\right) / \rho_{f}=0,10
\end{gathered}
$$

Avec $g=981 \mathrm{~cm} / \mathrm{s}^{2}$, nous aboutissons à :

$$
\Delta=1300 \overline{\mathrm{C}} \quad\left(\mathrm{cm}^{2} / \mathrm{s}^{3}\right)
$$

Considérons maintenant les paramètres de la dissipation visqueuse, pour la profondeur de $40 \mathrm{~cm}$, mais avec deux types d'agitation distincte.

$$
\begin{aligned}
& \text { a) } \mathrm{F}_{\mathrm{GLN}}=2 \mathrm{hz} \text { : } \\
& u^{\prime}=8,0 \mathrm{~cm} / \mathrm{s}, \quad \lambda=3 \mathrm{mn} \\
& \text { b) } \mathrm{F}_{\mathrm{GLN}}=6 \mathrm{hz} \text { : } \\
& u^{\prime}=17,5 \mathrm{~cm} / \mathrm{s}, \quad \lambda=1,5 \mathrm{mn}
\end{aligned}
$$

Avec la valeur de $\nu=10^{-2} \mathrm{~cm} / \mathrm{s}^{2}$, nous pouvons calculer la dissipation visqueuse dans les deux cas:

a) $\mathrm{F}_{\mathrm{GeN}}=2 \mathrm{hz}$

$$
15 \nu u^{\prime 2} / \lambda^{2}=0,15 \times 64 / 0,09=108 \mathrm{~cm}^{2} / \mathrm{s}^{2}
$$

b) $\mathrm{F}_{\mathrm{GEN}}=6 \mathrm{hz}$ :

$$
15 \vee u^{\prime 2} / \lambda^{2}=0,15 \times 306 / 0,022=2040 \mathrm{~cm}^{2} / \mathrm{s}^{2}
$$

En ce qui concerne la concentration moyenne, nous avons évalué qu’à la quantité maximum des billes dans la cuve $\left(\mathrm{C}_{\mathrm{ToT}}=3,6 \%\right)$ correspond, au fond de la cuve, une concentration locale comprise entre 10 et $15 \%$. Comme il ne s'agit que d'évaluer l'ordre de grandeur de l'effet des particules solides sur la turbulence, nous pouvons admettre que $\overline{\mathrm{C}}$ est de l'ordre de $10 \%$ (bien que $\overline{\mathrm{C}}$ soit un peu plus grand à ce niveau pour $F_{G S N}=2 \mathrm{hz}$ que pour $F_{G \mathrm{EN}}=6 \mathrm{hz}$ ). Nous avons donc, suivant l'expression qui donne la dissipation d'énergie, due à la présence des billes :

$$
\Delta=1300 \overline{\mathrm{C}}=130\left(\mathrm{~cm}^{2} / \mathrm{s}^{3}\right)
$$

Si nous considérons maintenant le rapport entre l'ordre de grandeur de cette dissipation, due aux billes, et la dissipation visqueuse directe, nous pouvons constater :

a) Pour les faibles intensités de turbulence $\left(\mathrm{F}_{\mathrm{GEN}}=2 \mathrm{hz}\right)$, $\Delta$ est du même ordre de grandeur que $15 v u_{1}^{\prime}{ }^{2} / \lambda_{1}{ }^{2}$ $(130 \longleftrightarrow 108)$. Le bilan de la dissipation est donc bien différent, de sorte que la turbulence est affectée par la présence des billes, et considérablement réduite.

b) Pour les fortes intensités de turbulence $\left(\mathrm{F}_{\mathrm{G} \mathrm{Ex}}=5 \mathrm{hz}\right)$, $\Delta$ est négligeable par rapport à $15 \vee u^{\prime}{ }_{1}{ }^{2} / \lambda_{1}{ }^{2}$. La structure de la turbulence, en présence des particules, n'est alors pratiquement pas modifiée par rapport à ce qu'elle est dans l'eau pure.

(*) Bien que, comme nous l'avons dit, elle ne prendrait en compte qu'une partie des effets des matériaux en suspension.

\section{Spectre d'énergie.}

Nous avons noté que les spectres d'énergie, avec et sans particules, n'étaient pas les mêmes. En particulier, l'énergie correspondant aux gros tourbillons était très notablement réduite en présence des particules, au profit de l'énergie contenue dans le tourbillon à faible dimension caractéristique, les modifications étant dans ce dernier cas relativement faibles. C'est une constatation importante, compte tenu des divergences de vue des différents auteurs à ce sujet. Mais Field [14] pense, lui, que «les particules infiuencent les tourbillons qui ont une échelle temporelle de même ordre de grandeur que la constante de temps des particules ».

C'est ce que nous allons qualitativement vérifier.

La constante de temps des billes a été déterminée à propos de mesures relatées dans [6], qui avaient pour but de déterminer la vitesse de chute en eau calme des billes ayant les mêmes caractéristiques physiques que celles utilisées ici. Nous avions alors trouvé :

$$
\tau=1,50 \mathrm{~s} .
$$

On peut maintenant déterminer la constante de temps des petits et des gros tourbillons.

Petits tourbillons - Ils sont caractérisés par l'échelle de temps de dissipation, $t_{d}$, telle que:

$$
t_{d}=\sqrt{\varepsilon / \nu}=\lambda / \sqrt{15} u^{\prime}
$$

(compte tenu de ce que $\varepsilon=15 \nu u^{\prime 2} / \lambda^{2}$ )

$$
\begin{aligned}
& \text { Pour } \mathrm{F}_{\mathrm{GJNX}}=2 \mathrm{hz}, \text { nous avons : } \\
& \qquad \begin{array}{c}
\lambda=4 \mathrm{~mm}, \quad \quad u^{\prime} \simeq 3,5 \mathrm{~cm} / \mathrm{s} \\
t_{d} \simeq \lambda / 4 u^{\prime}=0,028 \mathrm{~s}
\end{array}
\end{aligned}
$$

Ils ne seraient pratiquement pas influencés par les billes, sauf, comme nous le verrons, par transfert d'énergie, à partir des gros tourbillons dus au mouvement des billes.

Gros tourbillons - On peut évaluer leur temps caractéristique par l'échelle intégrale $\mathrm{L}$, et par la vitesse turbulente $u^{\prime}$. Donc :

$$
\mathrm{T}=\mathrm{L} / \mathrm{u}^{\prime}
$$

Dans le cadre des mesures de spectre que nous avons effectuées pour $F_{G E N}=2$ et $3 \mathrm{hz}$, nous avons abouti aux valeurs suivantes:

$$
\begin{gathered}
\mathrm{L}=6 \mathrm{~cm}, \quad u^{\prime}=3,5 \mathrm{~cm} / \mathrm{s} \\
\mathrm{T}=\mathrm{L} / u^{\prime}=6 / 3,5=1,7 \mathrm{~s}
\end{gathered}
$$

qui est bien du même ordre de grandeur que la constante de temps des particules.

Physiquement, cette influence des particules sur les tourbillons d'un temps caractéristique voisin de la constante de temps des particules pourrait être expliquée de la façon suivante. Assimilons les pulsations turbulentes à des mouvements harmoniques simples, caractérisées par un temps bien défini (inverse de la fréquence). Chaque pulsation - agissant indépendamment — tendrait à mettre les billes en mouvement alternatif, avec une certaine vitesse.

Pour des pulsations de temps caractéristiques décroissant, les billes suivraient d'abord le mouvement du fluide, puis 
l'écart de vitesse croîtrait, jusqu'à ce que les billes deviennent pratiquement immobiles pour des pulsations de temps caractéristiques réduits devant la constante de temps des billes. Le travail effectué par une pulsation de fréquence donnée (donc aux dépens de l'énergie de celle-ci) serait nul pour des pulsations à temps caractéristique élevé (la bille «suivrait» le fluide, et l'effort mutuel serait alors très faible), et à nouveau nul pour des pulsations à temps caractéristique très faible: Les billes étant pratiquement immobiles, le travail de ces pulsations serait nul. Mais ce travail - donc d'énergie dissipée - serait maximum lorsque le produit de l'effort mutuel bille-fluide par la vitesse absolue de la particule serait maximum, donc lorsque le temps caractéristique des pulsations et la constante de temps des billes seraient du mêne ordre de grandeur.

L'énergie dissipée par le mouvement relatif proviendrait donc surtout des pulsations à temps caractéristique voisines de la constante de temps des particules solides.

Parallèlement, au mouvement relatif billes-pulsation correspondrait un écoulement derrière les billes avec un sillage, donc des tourbillons de dimensions beaucoup plus réduites que ceux qui agissent sur la particule. Le mouvement relatif contribuerait ainsi directement au processus de cascade, en transférant directement l'énergie, à partir des tourbillons qui agissent sur les billes, vers les structures fines. L'énergie correspondant à celle-ci devrait bien alors être augmentée. C'est ce qui pourrait expliquer le léger accroissement d'énergie constatée dans les spectres, pour les structures fines de turbulence.

\section{Mesures au capteur de pression}

L'avantage de cette méthode sur l'utilisation du fil chaud, est dans notre cas évidente: le capteur est évidemment robuste et insensible aux heurts avec les corps en suspension. Mais elle présente aussi des inconvénients: La grandeur qu'on mesure n'est pas pure et bien définie comme dans le cas du fil chaud (la vitesse turbulente); elle est complexe et comprend des termes provenant des fluctuations de pression, des fluctuations de vitesse, et les corrélations entre ces deux valeurs. Dans le cadre de notre étude, l'incertitude qui en résulte est réduite dans une large mesure: nous déterminons avant tout des grandeurs mesurées avec et sans particules, et le rapport entre ces deux valcurs qui seul en fait nous intéresse, a une signification beaucoup plus profonde que les valeurs absolues.

La comparaison des mesures effectuées au fil chaud et au capteur n'est pas sans intérêt intrinsèque. Elle nous a confirmé, notamment lorsque nous avons pu appliquer successivement les deux méthodes, que les grandeurs mesurées avec le capteur de pression caractérisaient bien l'intensité de la turbulence.

\section{Principe. Mise au point de la méthode.}

Le schéma d'ensemble est donné à la figure 19 tandis que le résultat des mesures en eau pure est donné figure 20. On relève immédiatement que les mesures des fluctuations de pression et les mesures au fil chaud présentent une variation qualitative, en fonction de la profondeur, identique.

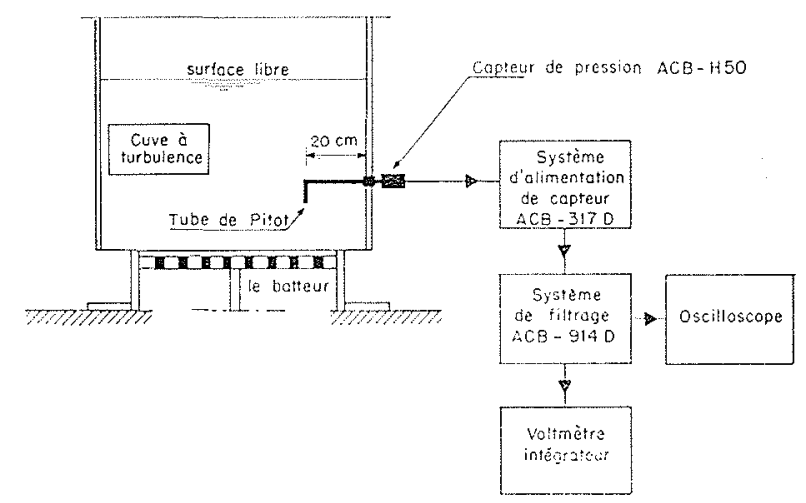

19/ Ensemble de mesure par la méthode de capteur de pression.

L'étude plus détaillée fait apparaître toutefois des phénomènes accessoires mais importants. L'analyse spectrale du signal met en évidence deux anomalies:

- La fréquence du batteur provoque l'apparition d'une énergie plus intense dans la bande voisine. En effet, au mouvement de la plaque sont associées de violentes fluctuations de pression dans la partie de la cuve comprise entre le fond et la plaque. Elles se transmettent ensuite dans l'ensemble de la cuve, et apparaissent alors dans l'analyse spectrale, à la fréquence, variable, du batteur.

- Le système tube de pitot-capteur présente une fréquence propre, au voisinage de $30 \mathrm{hz}$. Celle-ci est indépendante du mouvement du batteur. 21

Les résultats de l'analyse spectrale sont représentés figure

L'énergie contenue dans ces deux bandes est sans doute indépendante de celle contenue, aux mêmes fréquences, dans les pressions fluctuantes que mesure le capteur.

\section{Résultats et interprétation.}

Les relevés expérimentaux de la fluctuation de pression totale, en présence de billes, sont représentés figures 22 à 25 .

Là encore, les résultats des mesures au capteur sont cohérents avec les résultats de mesures au fil chaud, chaque fois que celles-ci ont pu être réalisées, donc quand la concentration en particules était assez faible (faibles fréquences du mouvement générateur, ou parties hautes de la cuve).

On remarque encore que :

Pour une faible turbulence $\left(\mathrm{F}_{\mathrm{GWN}}=3 \mathrm{hz}\right)$, les fluctuations de pressions sont partout réduites, sauf à la profondeur 40 . L'ordre de grandeur des coefficients de réduction est comparable à celui mesuré au fil chaud.

Pour de fortes turbulences $\left(\mathrm{F}_{\mathrm{GEN}}=4\right.$ et surtout $5 \mathrm{hz}$ ), la réduction des fluctuations de pression est plus faible, surtout pour $\mathrm{F}_{\mathrm{GiN}}=5 \mathrm{hz}$. Pour les fréquences 5 et $6 \mathrm{hz}$, le résultat s'inverse, et les fluctuations sont plus importantes aux profondeurs 30 et 40 quand il y a des matériaux en suspension.

Ce phénomène d'accroissement de l'énergie turbulente au fond est lié au principe utilisé pour créer la turbulence dans la cuve, et on peut considérer que l'énergie est plus grande pour trois raisons: 
a) L'énergie est plus grande du fait de l'augmentation moyenne des masses spécifiques.

$\mathrm{Si}$ les vitesses moyennes turbulentes étaient exactement les mêmes, l'énergie fluctuante moyenne serait de la forme $\mathrm{E}=\rho u^{\prime \prime}$, mais avec, en présence de particules:

$$
\mathrm{E}_{s}=\left[(1-\mathrm{C}) \mathrm{p}+\mathrm{C} \mathrm{p}^{\prime}\right] u^{\prime 2}
$$

où $\rho$ est la densité des particules solides et $c$ la concentration.

b) La plaque, pendant le mouvement, entraine les billes par contact direct. Rappelons que la vitesse maximum de la plaque est de l'ordre de $130 \mathrm{~cm} / \mathrm{s}$.

c) Lorsque la plaque descend, les billes sont lancées à travers les trous avec une assez grande vitesse (de l'ordre de $600 \mathrm{~cm} / \mathrm{s}$ ).

Dans les deux derniers cas, l'excès d'énergie correspondant à l'excédent de vitesse des particules ne peut qu'être transféré à l'eau, d'où l'augmentation apparente de l'énergie turbulente.

De toutes façons, si on confronte les résultats de la figure 25 avec les considérations évoquées au chapitre précédent, on constatera que, systématiquement, quel que soit le cas expérimental considéré, l'atténuation de la turbulence, à tous les niveaux, est plus grande en présence des particules. Tout se passe donc comme si, au bilan énergétique dans l'eau pure, se superposait un terme dû à l'influence des particules, qui se traduirait par une dissipation supplémentaire $\Delta$. C'est le terme que nous avons introduit dans l'équation d'énergie du chapitre précédent, en lui donnant comme expression approximative la valeur qui résultait du calcul de Barenblatt.

\section{Conclusions}

Les résultats de la cuve sont-ils transformables au cas d'une turbulence rencontrée dans un écoulement avec vitesse moyenne?

Evidemment, l'origine de la turbulence est dans ce cas bien différente. Dans notre cas, elle trouve son origine dans le mouvement de la plaque, et elle est ensuite transportée par diffusion sur toute la hauteur de la cuve. Dans le cas d'un écoulement à gradient de vitesse, la turbulence provient essentiellement, en un point, du travail des tensions de Reynolds, $\overline{u_{i} u_{m}}\left(\partial u_{i} / \partial x_{m}\right)$, et de la diffusion, par l'écoulement turbulent lui-même, de l'énergie turbulente engendrée par ces mêmes tensions, en un autre point, ce second terme étant en général faible devant le premier.

Le fait que l'origine de la turbulence soit plus locale et répartie ne nous paraît toutefois pas devoir modifier les conclusions que nous pourrions tirer des essais menés dans notre cuve: l'interprétation que nous avons donnée des phénomènes constatés n'amène pas à introduire l'origine de la turbulence.

Les résultats sont-ils extrapolables à des matériaux ou des écoulements ayant des caractéristiques différentes de celles envisagées ici?

Les paramètres du mouvement relatif fluide particule sont nombreux, si on introduit, comme il se doit, les paramètres fondamentaux de la turbulence. On devrait considérer en particulier les paramètres:

$\rho_{s} / \rho_{f}$ masse spécifique relative particule-fluide.

Un ou plusieurs paramètres représentant les dimensions relatives de la turbulence par rapport aux dimensions des particules: $d / \eta, d / \lambda, d / \mathrm{L}$ qui comparent le diamètre des particules à l'échelle de dissipation, la micro échelle et l'échelle intégrale. Ces longueurs ne sont pas indépendantes, puisque leurs rapports sont fonctions du nombre de Reynolds de la turbulence.

Un ou plusieurs temps caractéristiques de la turbulence:

$$
\tau / \tau_{d} \quad \tau / \mathrm{T}
$$

$\tau_{d}$ représentant l'échelle de temps de dissipation, de Kolmogoroff, $\mathrm{T}$ l'échelle intégrale, et $\tau$ la constante de temps des particules.

Bien entendu, on aurait à considérer aussi la concentration en matériaux solides.

Les valeurs extrêmes des uns ou des autres de ces paramètres correspondent à des cas bien différents :

Si :

$$
\rho_{s} / \rho_{f} \# 1, \quad d / \eta \ll 1, \quad \tau / \tau_{d} \ll 1
$$

les particules suivent le mouvement du fluide, et l'ensemble constitue un milieu quasi homogène.

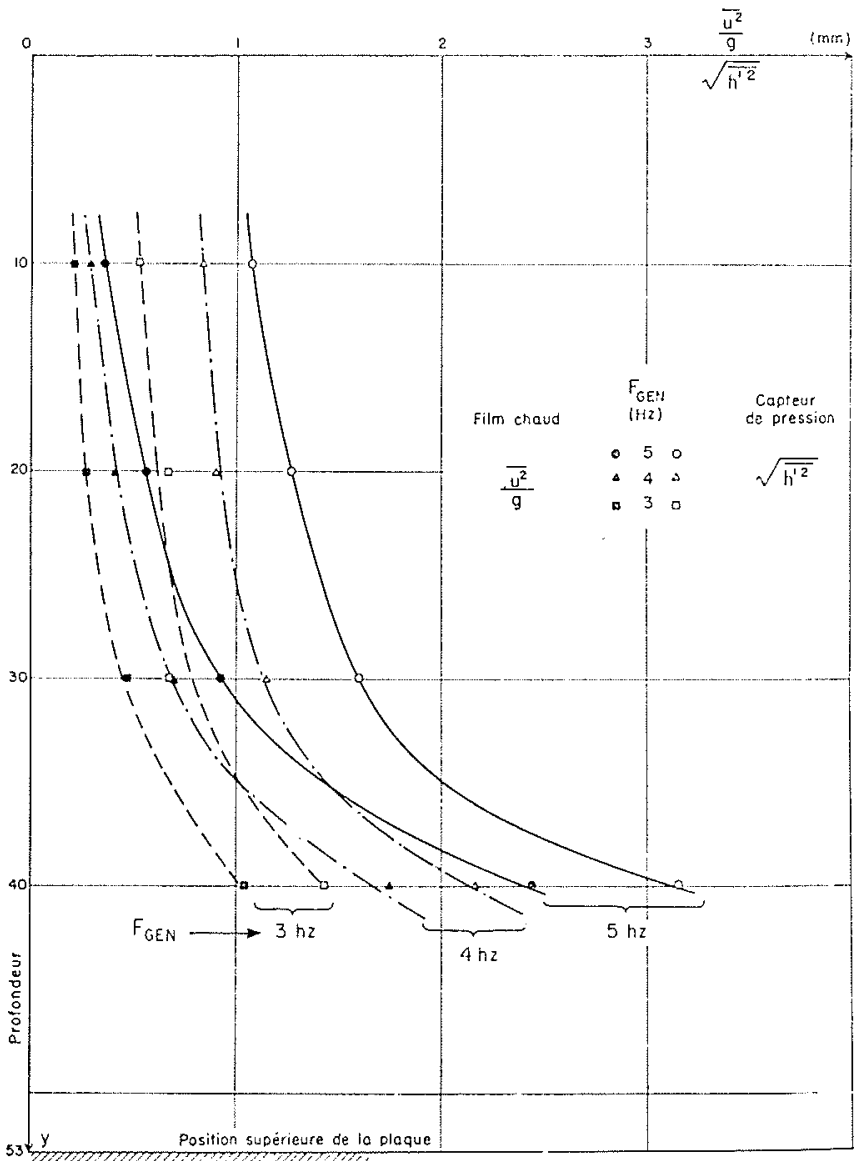

20/ Comparaison des mesures au fil chaud et au capteur de pression. 


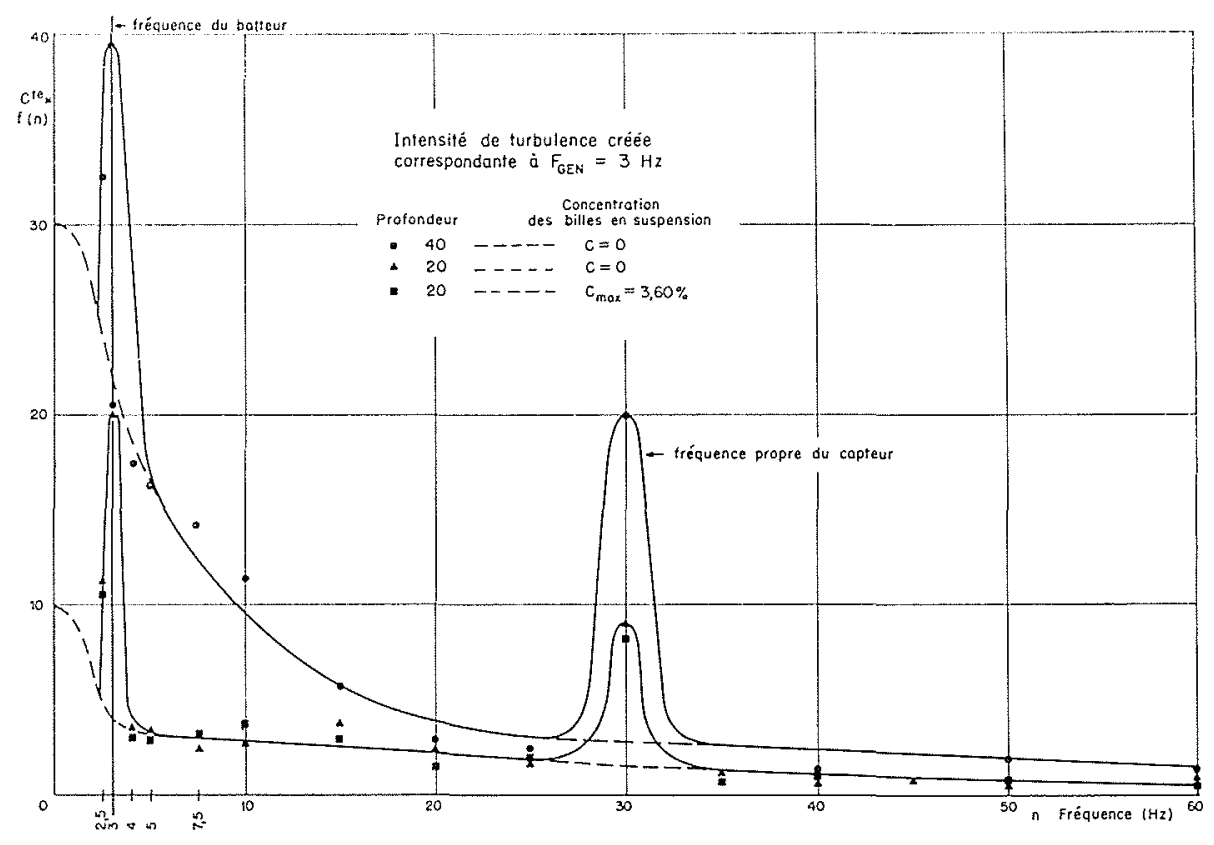

21/ Spectres (analyse spectrale des signaux du capteur de pression).

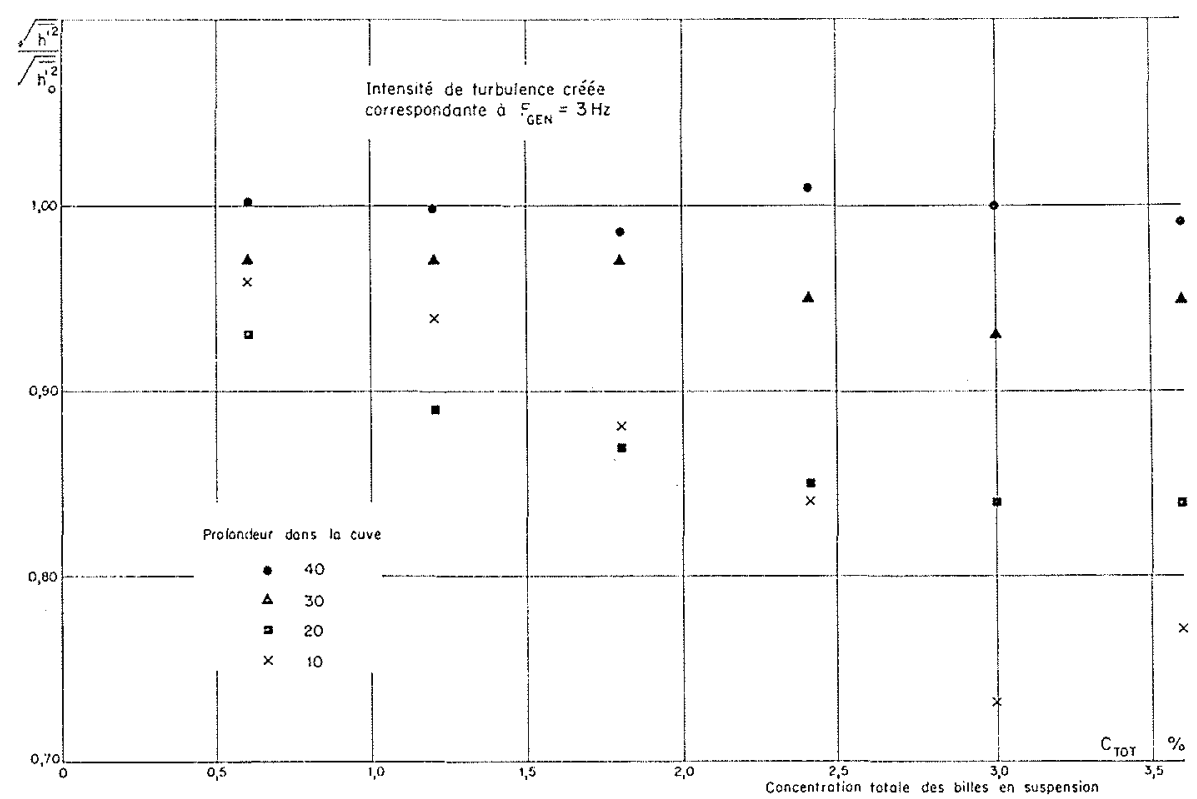

22/ Réduction des grandeurs mesurées au capteur de pression par rapport à l'eau pure en présence des particules solides en suspension. 


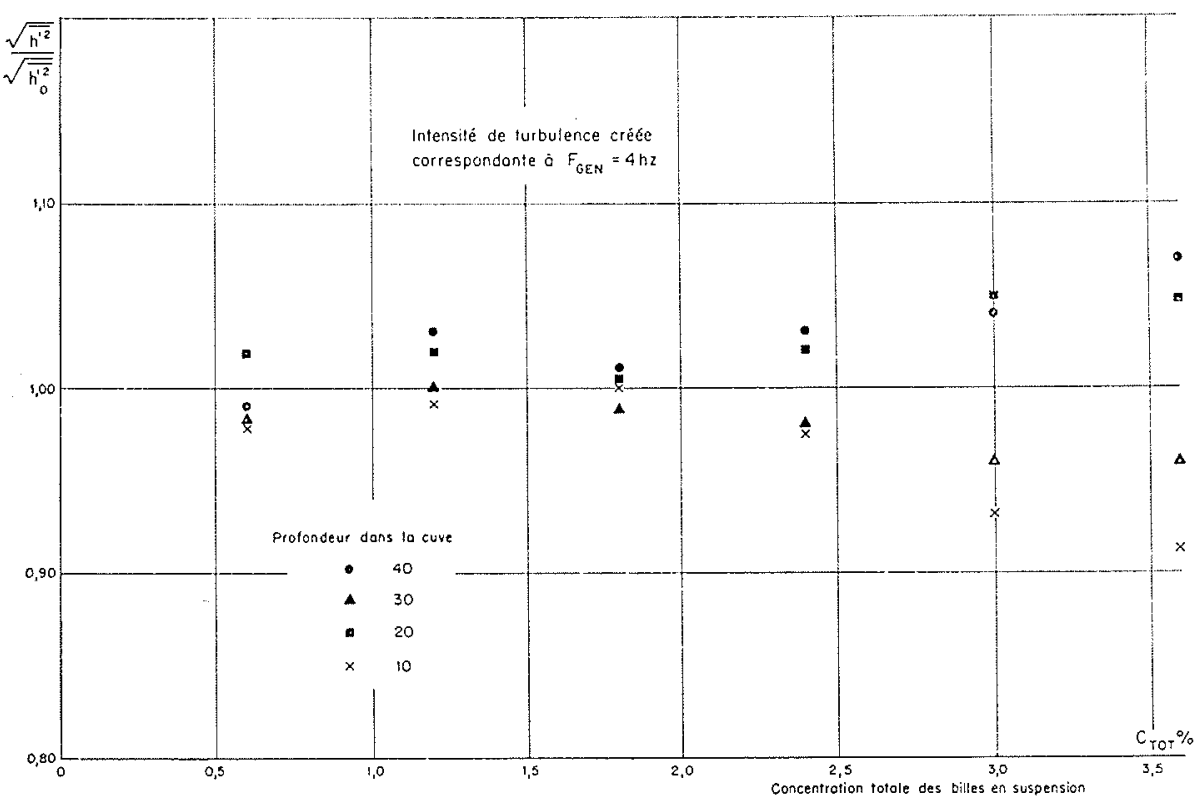

23/ Réduction des grandeurs mesurées au capteur de pression par rapport à l'eau pure en présence des particules solides en suspension.

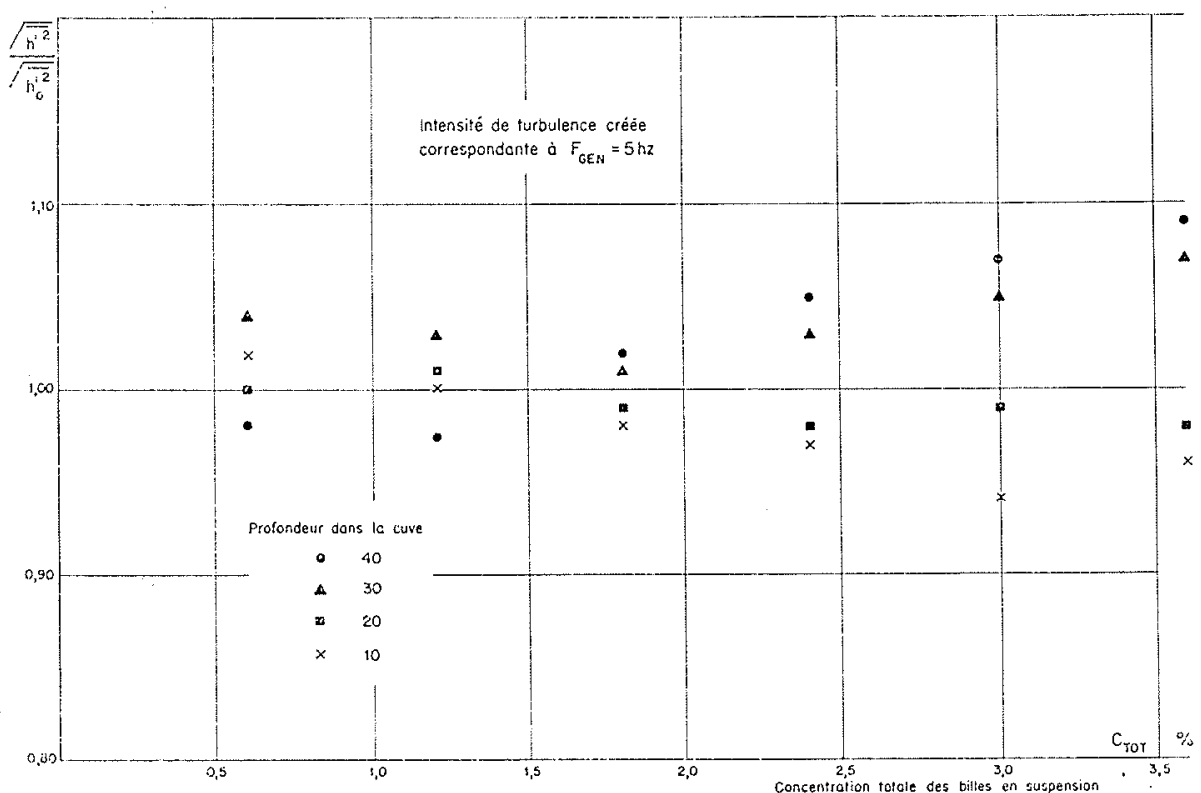

24/ Réduction des grandeurs mesurées au capteur de pression par rapport à l'eau pure en présence des particules solides en suspension. 


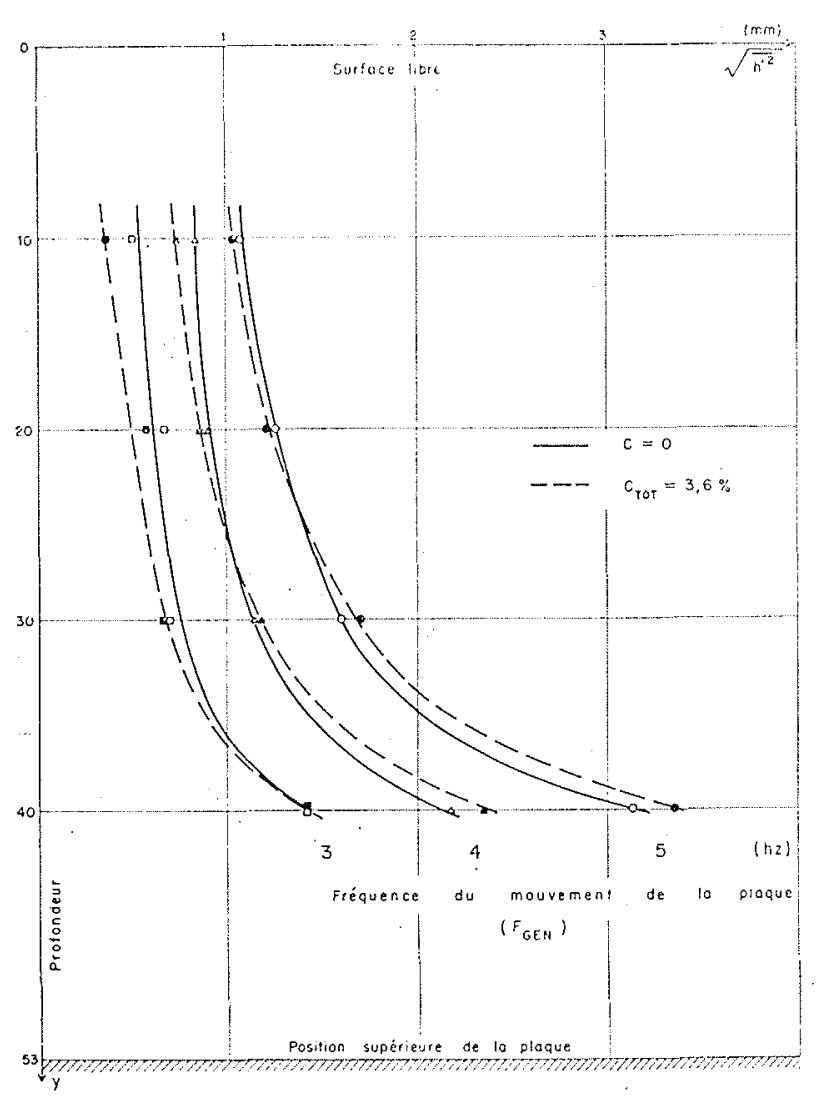

25/ Modification des grandeurs mesurées au capteur de pression sous l'influence de particules solides en suspension.

Si, au contraire :

$$
\rho_{s} / \varphi_{f} \# 1, \quad d / \eta \gg 1, \quad \tau / \tau_{d} \gg 1
$$

les particules sont en mouvement relatif par rapport au fluide.

Les conditions de notre expérience correspondent à ce dernier cas.

Sous réserve de leur limitation au cas où les conditions expérimentales sont de même nature, les conclusions suivantes se dégageraient des essais et de l'analyse qui en a été facite.

- La présence des matériaux solides en suspension réduit la turbulence du milieu porteur, par rapport à ce qu'elle serait sans matériaux.

- Cette réduction est d'autant plus grande que la concentration en matériaux est plus élevée.

- Cette influence est «sensible», c'est-à-dire que, même pour des concentrations faibles, on obtient une réduction substantielle de la turbulence, en particulier si elle est peu intense.
On pourrait tenter d'appliquer ces conclusions au transport des matériaux par une veine fluide, par exemple un écoulement à surface libre, plan et uniforme. La turbulence est alors maximum sur le fond, et caractérisée par la vitesse de frottement $u_{*}$.

- Un écoulement de cette nature serait en mesure de transporter en suspension des matériaux ayant une vitesse de chute en eau calme inférieure (ou d'un ordre de grandeur voisin) à la vitesse turbulente au fond, elle-même de l'ordre $u_{*}$.

- La charge en matériaux en suspension réduirait la turbulence au fond, qui serait ainsi une fonction décroissante de la concentration. La valeur limite de la concentration serait celle qui rendrait la vitesse turbulente au fond voisine de la vitesse de chute en eau calme du matériau considéré.

- La charge maximum en matériaux d'un écoulement serait fonction de l'écart entre la vitesse turbulente au fond, et la vitesse de chute en eau calme, soit $u * / W$. La concentration de saturation croîtrait rapidement en fonction de ce rapport.

\section{Bibliographie}

[1] Craya (A.). - Introduction à la théorie de la turbulence. Séminaire de turbulence, Académie des sciences de Pologne, Jablona (1961).

[2] Bouvard (M.) et Dumas (H.). - Application de la méthode du fil chaud à la mesure de la turbulence dans l'eau. La Houille Blanche, $\mathrm{n}^{\circ} 3$ et 7, 1967.

[3] Bouvard (M.). - Considérations théoriques sur les matériaux en suspension des particules solides dans un courant turbulent uniforme. Mémoires et travaux de la Société Hydrotechnique de France, La Houille Blanche, n" spécial B 1955.

[4] Bouvard (M.). - Effet d'inertie dans le transport en suspension. Essal de détermination de la saturation en suspension d'un écoulement. Congrès AlRH, Montréal (1959).

[5] BarenblatT (G. J.). - Sur le mouvement des particules en suspension dans un écoulement turbulent. Extrait du Préklad Mat i Melih, t. XVII (mai 1953).

[6] Bouvard (M.). - Turbulence sans vitesse moyenne et diffusion des particules solides dans l'eau. Thèse d'Etat, Grenoble, 1967.

[7] Fortier (A.). - Mécanique des suspensions. Masson, Paris (1967).

[8] Hinze. - Turbulence. Mc Graw Hill Book Company (1959).

[9] Hivo (M.). - Turbulent flow with suspended particles. Proceeding ASCE (juillet 1963).

[10] Soo (J. L.). - Fluid Dynamics of multiphase systems. Blaisdele Publishing Company, London (1967).

[11] Vasileve (D. F.). - Problem of two Phase Flow theory. Gene ral lecture, XIII ${ }^{\circ}$ congrès AIRH, Kyoto, Japon (1969).

[12] Frankle. - Divers compte rendus à l'Académie des Sciences de l'U.R.S.S., et notamment: On the system of equations of suspended sediment motion. 1960; Academy of Sciences of the USSR, pp. 132-137 (cf. VAsILIEv, Problems of two Phases flow theory; General report, AIRH, Kyoto, 1969).

[13] VANoni et Nomicos. - Resistance properties of sediment-laden streams. Transactions ASCE, vol. 125 (1960).

[14] FiELD. - Entraînement into an air jet laden with particles. Circulaire 272, du «British Coal Utilisation Research Association $\gg(1963)$. 